\title{
Radiation- and pair-loaded shocks
}

\author{
Maxim Lyutikov \\ Department of Physics and Astronomy, Purdue University, \\ 525 Northwestern Avenue, West Lafayette, IN 47907-2036
}

\begin{abstract}
We consider the structure of mildly relativistic shocks in dense media, taking into account the radiation and pair loading, and diffusive radiation energy transfer within the flow. For increasing shock velocity (increasing post-shock temperature), the first important effect is the efficient energy redistribution by radiation within the shock that leads to the appearance of an isothermal jump, whereby the flow reaches the finial state through a discontinuous isothermal transition. The isothermal jump, on scales much smaller that the photon diffusion length, consists of a weak shock and a quick relaxation to the isothermal conditions. Highly radiation-dominated shocks do not form isothermal jump. Pair production can mildly increase the overall shock compression ratio to $\approx 10$ (from 4 for matter-dominated shocks and 7 of the radiation-dominated shocks).
\end{abstract}

Subject headings: shock waves; relativistic processes; plasmas; radiation: dynamics

\section{Introduction}

In many astrophysical settings shocks propagate through dense environment with mildly relativistic velocities, so that the post-shock radiation pressure can exceed the thermal pressure (Budnik et al. 2010). In addition, astrophysical shocks may heat up the plasma to temperatures where postshock pair thermal production may become important. For example, the discovery of GRB170817A associated with gravitational waves event GW170817 (Abbott et al. 2017) is best explained due to the emission from the jet propagating with mildly relativistic velocities through a dense wind generated by the accretion torus (Lazzati et al. 2017; Pozanenko et al. 2017; Gottlieb et al. 2017; Bromberg et al. 2017). In this paper we address the structure of pair and radiation loaded shock transitions.

Several non-standard phenomena are expected in these types of shocks. First, a large fraction of the energy flux can be converted into radiation and pairs, thus modifying the thermodynamic properties of plasma by both contributing to effective mass density and to pressure. Secondly, energy redistribution within the shock may have profound influence on the shock properties. Especially important is photon diffusion - even if the energy density in photons is below the thermal energy density, the high photon mean free path may modify the shock considerably (Zeldovich \& Raizer 2003). 
One of the key features of the radiation-modified shocks is the appearance of an isothermal discontinuity (Rayleigh 1910; Landau \& Lifshitz 1959; Zeldovich \& Raizer 2003). As the incoming flow is decelerated and compressed by scattering of the incoming radiation, its temperature and compression ration increase. For sufficiently strong shocks the final temperature is reached before the final compression ratio. As a result, an isothermal jump forms.

Appearance of the isothermal discontinuity in relativistic shocks have been previously considered by Chapline \& Granik (1984), though they did not take into account pair production. Here we take pair production fully into account.

We assume that plasma is sufficiently dense so that radiation is trapped within the flow. The first most important effect is the diffusion of radiation - even if energy density and mass loading due to radiation (and of pairs) are small comparable to matter energy density, the redistribution of energy within the shocked flow is important. This leads to the formation of isothermal jump, Landau \& Lifshitz (1959); Zeldovich \& Raizer (2003) and $\S 4$. (To clarify the notation, the isothermal jump is different from highly radiatively cooled isothermal shocks; in our case the flow is energy conserving.) On the other hand, for very strong highly radiation-dominated shocks the pressure created by photons and pairs may dominate over the ion pressure. This introduces further complications: the isothermal jump can disappear, Zeldovich \& Raizer (2003) and $\S 5$.

\section{Protons, radiation and pairs}

\subsection{Protons, radiation and pairs at relativistic temperatures}

In what follows we normalize the temperature to the electron temperature

$$
\theta \equiv T /\left(m_{e} c^{2}\right)
$$

and the number $n$ density to the dimensionless $\tilde{n}$

$$
n \equiv \tilde{n} / \lambda_{C}^{3}
$$

where $\lambda_{C}=\hbar /\left(m_{e} c\right)$ is the Compton wavelength. Dimensionless number density of 1 corresponds to

$$
\begin{aligned}
& n=1 / \lambda_{C}^{3}=1.7 \times 10^{31} \mathrm{~cm}^{-3} \\
& \rho=m_{p} n=2.8 \times 10^{7} \mathrm{~g} \mathrm{~cm}^{-3}
\end{aligned}
$$

Such densities are expected in the region near the accretion shock during core collapse (Mezzacappa et al. 2001) and in the tidal tails/wind generated during neutron star mergers (e.g. Metzger et al. 2010). For example, in the case of neutron star merger, a fraction of up to $10^{-2} M_{\odot}$ is injected with mildly relativistic velocities $v_{w} \sim 0.1 c$ for a duration $\approx 1$ second. The corresponding dimensionless density evaluates to $\tilde{n} \sim 10^{-5}$. (Below we drop the tilde sign over the dimensionless density). 
Consider next three contributions to the total enthalpy of plasma - from thermal motion of ions $w_{p}$, radiation $w_{r}$ and pairs $w_{ \pm}$(defined per unit volume). For plasma ions and radiation, the enthalpy can be written as

$$
\begin{aligned}
\frac{\lambda_{C}^{3}}{c^{2} m_{e}} w_{p} & =\frac{\gamma \theta n}{\gamma-1} \\
\frac{\lambda_{C}^{3}}{c^{2} m_{e}} w_{r} & =\frac{4 \pi \theta^{4}}{45}
\end{aligned}
$$

The pair pressure, enthalpy and number density is a fairly complicated function of temperature, especially in a weakly relativistic regime (e.g. Wandel \& Yahil 1979; Svensson 1984, and reference there in). To simplify the consideration, we adopt the following parametrization

$$
\begin{aligned}
& \frac{\lambda_{C}^{3}}{c^{2} m_{e}} w_{ \pm}=\frac{\sqrt{2} g_{E} e^{-1 / \theta} \sqrt{\theta^{3}}(4 \theta+1)}{\pi^{3 / 2}} \\
& g_{E}=1+0.47 \theta+0.37 \sqrt{\theta}+\sqrt{\frac{2}{\pi}} \theta^{3 / 2} \zeta(3),
\end{aligned}
$$

see Wandel \& Yahil (1979); Svensson (1984), $\zeta$ is the zeta-function (thus, pairs are assumed to be relativistic, factor of $4 \theta$ above). We expect that these simplifications for the pairs equation of state introduce only mild corrections.

For plasma with ion number density $n$ (normalized to $\lambda_{C}^{-3}$ ) the ratios of ion mass density to that of pairs is (for $\theta \leq 1$ )

$$
\frac{m_{p} n}{m_{e} n_{ \pm}}=\frac{\pi^{3 / 2}}{\sqrt{2}} \frac{e^{1 / \theta}}{\theta^{3 / 2}} \mu n
$$

where $\mu=m_{p} / m_{e}$. So that pairs dominate ions by mass for

$$
n \leq \frac{\sqrt{2}}{\pi^{3 / 2}} e^{-1 / \theta} \theta^{3 / 2} \mu^{-1}=5 \times 10^{-5}
$$

where the last equation assumes $\theta=1$. (In physical units this corresponds to density of $\rho \sim 1.4 \times 10^{3}$ $\mathrm{g} \mathrm{cm}^{-3}$.

The ratios of ion mass density to that of radiation is

$$
\begin{aligned}
& \frac{m_{p} n}{u_{\text {rad }} / c^{2}}=\frac{15}{\pi} \frac{\mu n}{\theta^{4}} \\
& u_{\text {rad }}=\frac{4}{c} \sigma_{S B} T^{4} \equiv \frac{\pi}{15} \theta^{4}
\end{aligned}
$$

Thus the radiation mass-density dominates the ion density at much lower $n$,

$$
n \leq \frac{\pi}{15} \frac{\theta^{3}}{\mu}
$$

(corresponding density is $\rho \sim 3.2 \times 10^{3} \mathrm{~g} \mathrm{~cm}^{-3}$ at $\theta=1$. 
The ratio of the ion pressure to radiation pressure is

$$
\frac{n T}{u_{\text {rad }} / 3}=\frac{45}{\pi} \frac{n}{\theta^{3}}
$$

so that for

$$
n \leq \frac{\pi}{15} \theta^{3}
$$

the radiation pressure dominates the ion pressure (for $\rho<2 \times 10^{6} \mathrm{~g} \mathrm{~cm}^{-3}$ ).

The pressure contribution of the radiation $p_{r}=u_{\text {rad }} / 3$ becomes dominate over matter contribution at mush higher densities:

$$
\frac{n T}{p_{r}}=\frac{45}{\pi} \frac{n}{\theta^{3}}
$$

so that pairs dominate ions by mass for

$$
n \leq \frac{\pi}{45} \theta^{3}
$$

(this evaluates to $\rho=2 \times 10^{6} \mathrm{~g} \mathrm{~cm}^{-3}$ for $\theta=1$ ).

\subsection{Photons and pairs at mildly relativistic shocks}

The relations discussed above can be adopted to shock-heated plasma. In this case for matterdominated regime

$$
\theta \approx 2 \frac{\gamma-1}{(1+\gamma)^{2}} \mu \beta_{1}^{2}=\frac{3}{16} \mu \beta_{1}^{2}
$$

If the upstream plasma has density $n_{1}$, the post-shock ion pressure become smaller than radiation pressure at

$$
\frac{2 \mu n_{1} \beta_{1}^{2}}{\gamma+1}<\frac{\pi}{45} \theta^{4} \rightarrow n<\frac{8 \pi(\gamma-1)^{4}}{45(\gamma+1)^{7}} \beta_{1}^{6} \mu^{3}
$$

In such highly radiation-dominated shock without pairs (still, mass density is dominated by ions)

$$
\begin{aligned}
& \eta=1 / 7 \\
& \theta_{\max }=\left(\frac{270}{7 \pi}\right)^{1 / 4} n_{1}^{1 / 4} \mu^{1 / 4} \beta_{1}^{1 / 2}
\end{aligned}
$$

Also, in the high compressibility limit $\eta \rightarrow 0$

$$
\theta=\left(\frac{45}{\pi}\right)^{1 / 4} n_{1}^{1 / 4} \mu^{1 / 4} \beta_{1}^{1 / 2}
$$

which is very close to (16).

For relativistic temperatures the condition (15) is also approximately the condition for pair pressure to dominate over ion kinetic pressure, while at $\theta \leq 1$ the post-shock ion pressure become 
smaller than pair pressure for

$$
n_{1} \leq \frac{4 \beta_{1}^{3}(\gamma-1)^{5 / 2} \mu^{3 / 2} e^{-\frac{(\gamma+1)^{2}}{2 \beta_{1}^{2}(\gamma-1) \mu}}}{\pi^{3 / 2}(\gamma+1)^{4}}
$$

Due to exponential dependence this ratio varies over large values at mild shock velocities.

Rewriting Eq. (15) as a condition on velocity, the post-shock radiation domination requires

$$
\beta>\left(\frac{60}{\pi}\right)^{1 / 6} n^{1 / 6} \mu^{-1 / 2}
$$

The post-shock temperature becomes $\theta>1$ at

$$
\beta \geq \frac{1}{\sqrt{\mu}} \sqrt{1+\frac{\pi}{60 n}}
$$

(this relation is valid for both matter and radiation-dominated shocks), see Fig. 1

\section{Stationary 1D flow with radiation and pair production}

Let's assume that a shock propagates with velocity $\beta_{1} \leq 1$ through a cold plasma with ion density $\rho_{1}$. At each point one-dimensional, stationary non-relativistic flow is described by the following set of equations (mass, momentum and energy flux conservation)

$$
\begin{aligned}
& \beta_{1} \rho_{1}=\beta \rho \\
& \rho_{1} \beta_{1}^{2}=p_{t o t}+\rho_{t o t} \beta^{2} \\
& \rho_{1} \beta_{1}^{3} / 2=\left(w_{t o t}+\rho_{t o t} \beta^{2} / 2\right) \beta+F_{r}
\end{aligned}
$$

where subscript 1 indicates quantities far upstream.

In $(21)$

$$
\rho_{\text {tot }}=\rho+n_{ \pm} m_{e}+u_{\text {rad }} / c^{2}
$$

is the total mass density that includes ion density $\rho$, pair mass density $n_{ \pm} m_{e}$ and effective mass density of radiation $u_{\text {rad }} / c^{2}$ ( $u_{\text {rad }}$ is the energy-density of thermal radiation),

$$
w_{2}=\frac{4}{3} u_{r a d}+\frac{\gamma}{\gamma-1} \frac{\rho}{m_{p}} T+n_{ \pm}\left(m_{e} c^{2}+4 T\right)
$$

is the plasma enthalpy not including ion rest mass, that includes contribution from radiation, ion energy density, pair rest mass and pair energy density, $\gamma=5 / 3$ is the adiabatic index of the ion component (assumed non-relativistic), the electron component is assumed to be relativistic (factor 


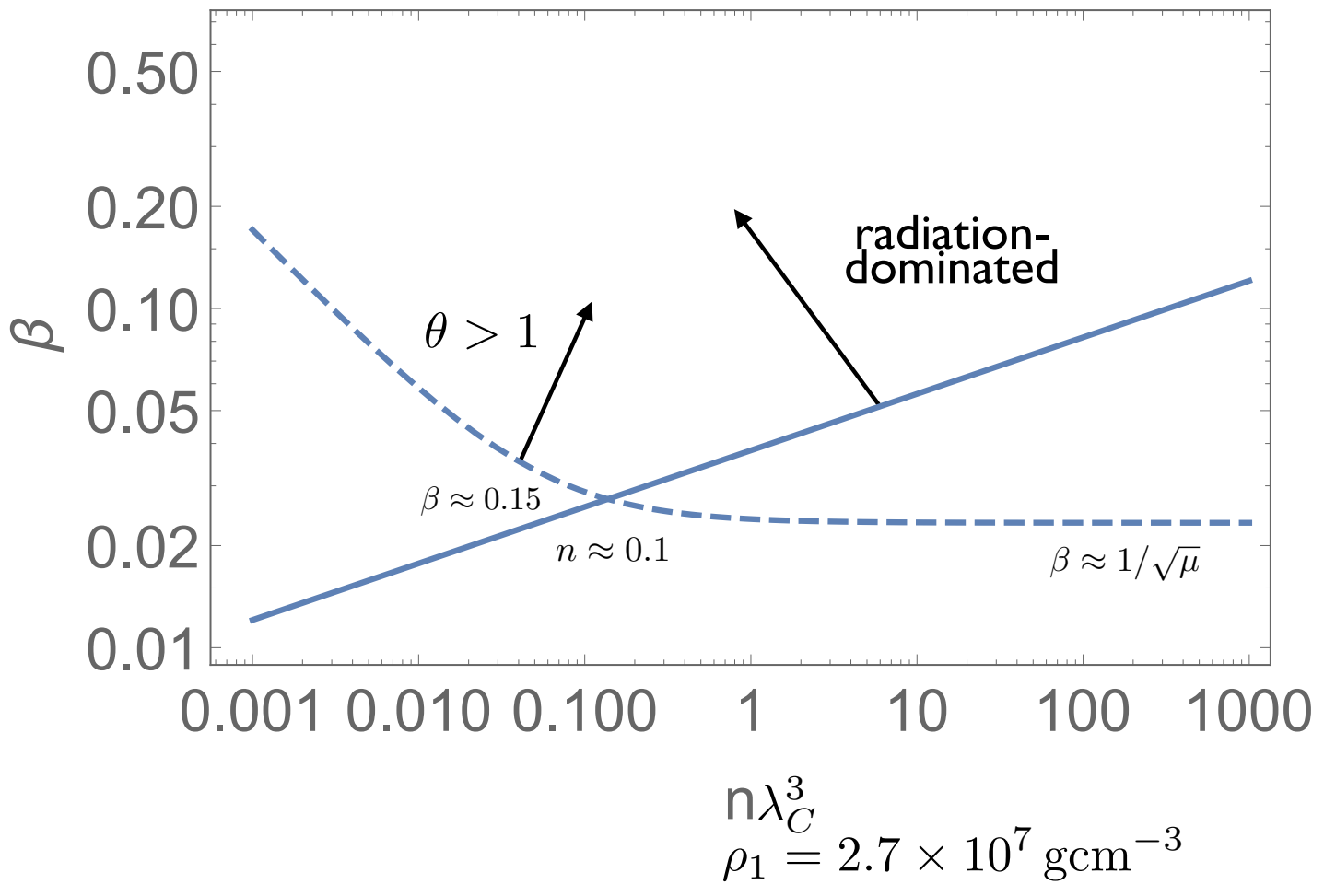

Fig. 1.- Regime of radiation-dominated shocks and relativistic post-shock temperatures on the $\beta-n$ diagram, see Eqns. (19) and (20)

of 4 in the expression for the pair contribution). Finally, $F_{r}$ is the energy flux carried by radiation. We assume high optical depth limit, so that locally

$$
\begin{aligned}
& F_{r}=-\frac{c}{3 n_{t o t} \sigma_{T}} \nabla u_{r a d} \\
& u_{\text {rad }}=\frac{4}{c} \sigma_{S B} T^{4}
\end{aligned}
$$

where total density has contributions both from neutralizing electrons and pairs, $n_{\text {tot }}=\rho / m_{p}+n_{ \pm}$.

\section{The first effect: formation of isothermal jump in strong shocks}

As the velocity of the shock grows, the energy redistribution within the flow by radiation becomes the first important effect - even if the radiation pressure is much smaller than the matter 
pressure. Let's neglect radiation energy density and pressure, but keep energy diffusion due to photons with large mean free path ( $c f$. Zeldovich \& Raizer 2003). Everywhere along the stationary flow we have conservation of matter, momentum and energy flux:

$$
\begin{aligned}
& \beta_{1} \rho_{1}=\beta \rho \\
& \rho_{1} \beta_{1}^{2}=\frac{\rho}{m_{p}} T+\rho \beta^{2} \\
& \rho_{1} \beta_{1}^{3} / 2=\left(\frac{\gamma}{\gamma-1} \frac{\rho}{m_{p}} T+\rho \beta^{2} / 2\right) \beta+F_{r}
\end{aligned}
$$

Introducing the inverse of the compression ratio $\eta=n_{1} / n$, Eq. (25) becomes

$$
\begin{aligned}
& \theta=\mu \beta_{1}^{2}(1-\eta) \eta \\
& \frac{\lambda_{C}}{15 \beta_{1} n_{1}^{2} \alpha_{f}^{2}} \partial_{x} \theta=\frac{\beta_{1}^{2}\left(\eta^{2}-1\right) \mu+\frac{2 \gamma \theta}{\gamma-1}}{\eta \theta^{3}}
\end{aligned}
$$

Far downstream temperature becomes constant, $\partial_{x} \theta=0$, and (26-27) give shock jump conditions

$$
\begin{aligned}
\eta_{2} & =\frac{\gamma-1}{\gamma+1} \\
\theta_{2} & =\frac{2 \beta_{1}^{2}(\gamma-1) \mu}{(\gamma+1)^{2}}
\end{aligned}
$$

Importantly, in order to reach this final state a flow should develop a special type of discontinuity - isothermal jump (Landau \& Lifshitz 1959; Zeldovich \& Raizer 2003). The isothermal jump in this case forms regardless of a particular form of $F_{r}$, as we discus next.

The momentum conservation (26) can also be written for the evolution of compression as a function of temperature $\eta(\theta)$

$$
\begin{aligned}
& \eta=\frac{1}{2}\left(1 \pm \sqrt{1-\frac{4 \theta}{\beta_{1}^{2} \mu}}\right)=\frac{1}{2}\left(1 \pm \sqrt{1-\frac{\theta}{\theta_{\max }}}\right) \\
& \theta_{\max }=\frac{\beta_{1}^{2} \mu}{4}
\end{aligned}
$$

Thus, there are two branches for $\eta(\theta)$; they connect at the point

$$
\begin{aligned}
& \eta_{\text {crit }}=\frac{1}{2} \\
& \theta_{\max }=\frac{\beta_{1}^{2} \mu}{4}
\end{aligned}
$$

Importantly, the initial point $\eta=1$ is on the upper branch, while the final jump conditions is on the lower branch. Yet the flow cannot pass continuously from the initial to the final point - using (26) the equation for the compression ratio becomes

$$
\frac{\pi \beta_{1}^{5}(\gamma-1) \mu^{3}}{15 \alpha_{f}^{2} n_{1}^{2} \lambda_{C}^{5}} \partial_{x} \eta=\frac{\gamma(\eta-1)+\eta+1}{(1-2 \eta)(1-\eta)^{2} \eta^{4}}
$$


Equation (31) clearly shows that the final state of $\eta=\eta_{2}=1 / 4$ (for $\gamma=5 / 3$ ) cannot be reached continuously: in order to reach it continuously one must pass through a special point $\eta=1 / 2$. This statement is true for any non-zero left hand side.

Note, that

$$
\frac{\theta_{2}}{\theta_{\max }}=8 \frac{\gamma-1}{(\gamma+1)^{2}}=\frac{3}{4}<1
$$

Thus, as the state evolves along the upper branch, the terminal temperature is reached before the the terminal compression. It is required that temperature increase monotonically (e.g. Landau \& Lifshitz 1959, Eq. (95.3)). Thus, since $\theta_{2}<\theta_{\max }$, the final state cannot be reached continuously. There should be an isothermal jump at $\theta=\theta_{2}$, Fig. (2). ${ }^{1}$

\subsection{Structure of the precursor}

In fact we can find an exact analytical solution of (31). Let us normalize out, by the precursor scale

$$
L_{p}=\frac{15 \alpha_{f}^{2} n_{1}^{2} \lambda_{C}^{5}}{\pi \beta_{1}^{5}(\gamma-1) \mu^{3}}
$$

The precursor scale $L_{p}$ is larger than the mean free path $\lambda_{m f p}=1 /\left(\sigma_{T} n_{1}\right)$,

$$
\frac{L_{p}}{\lambda_{m f p}}=\frac{8}{45} \pi^{2} \beta_{1}^{5}(\gamma-1) \mu^{3}
$$

for $\beta \geq 10^{-2}$. We find

$$
\partial_{x} \eta=\frac{\gamma(\eta-1)+\eta+1}{(1-2 \eta)(1-\eta)^{2} \eta^{4}}
$$

which has an analytical solution for $x(\eta)$ with boundary condition $x(1)=0$, Fig. 3 (temperature is related to compression ratio via (29) along the upper branch). Eq. (36) describes the precursor of the isothermal transition under assumption that energy density and pressure of pairs and radiation can be neglected, yet radiative flux diffusively redistribues energy within a flow.

Near $x \approx 0$ the compression ratio and temperature is $\eta \propto x^{1 / 3}$ (so that $d \eta / d x$ diverges). This is a drawback of the neglect of the upstream temperature; finite shock Mach number leads to the appearance of an exponential precursor, Appendix A.

\footnotetext{
${ }^{1}$ The local Mach number is

$$
M=\sqrt{\frac{\eta}{\gamma(1-\eta)}},
$$

it equals unity at $\eta_{M=1}=\gamma /(1+\gamma)=5 / 8$ (at this point $\left.\theta=\gamma /(\gamma+1) \mu \beta_{1}^{2}=(15 / 64) \mu \beta_{1}^{2}\right)$.
} 


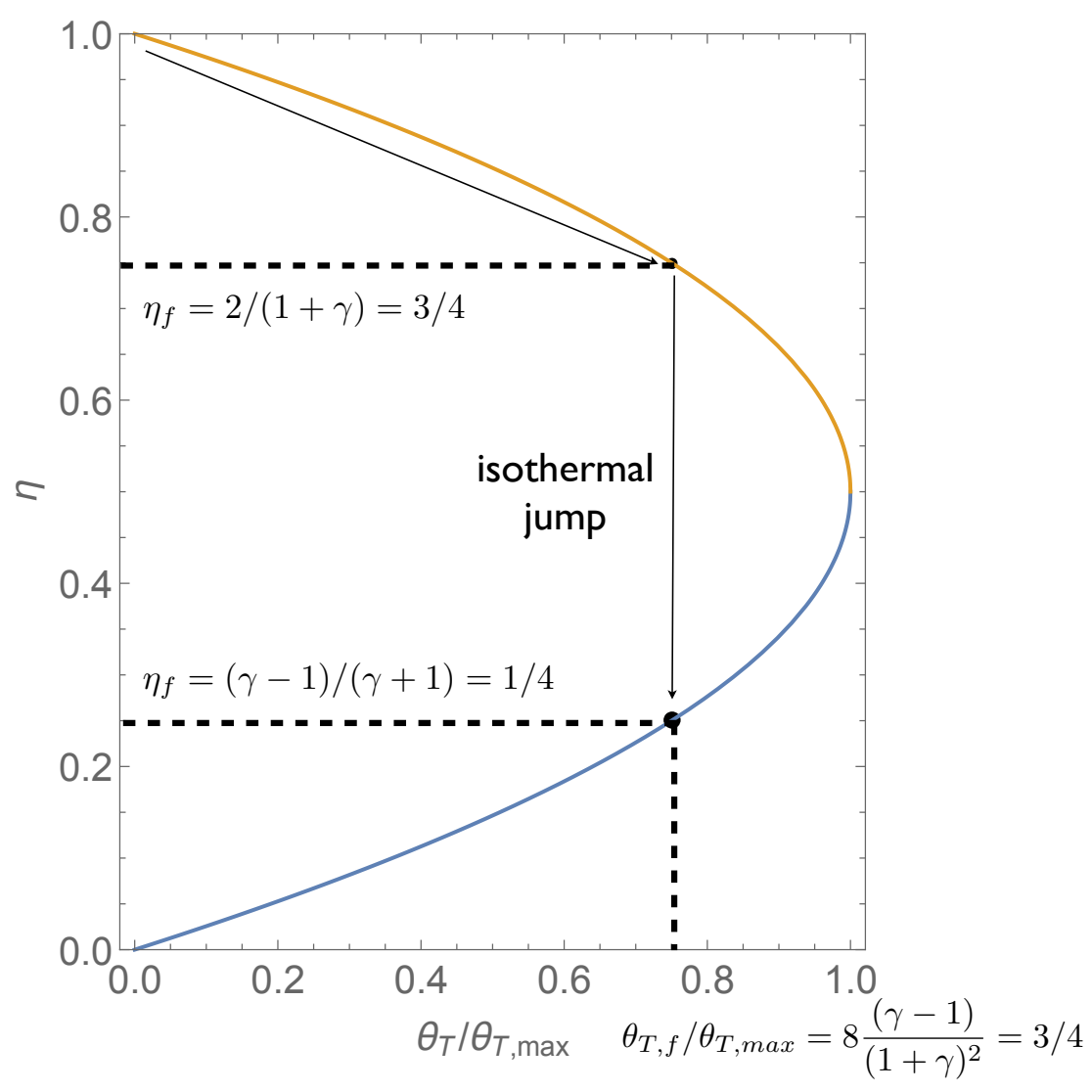

Fig. 2.- Evolution of the initially cold flow within the radiatively-mediated shock in the compression ratio $\eta$ - temperature $\theta$ plane neglecting momentum flux of pairs and radiation. The flow starts at $\eta=1, \theta=0$ and reaches the finite temperature $\theta_{2}$ at the moment when the inverse compression ratio $\eta_{+}$is smaller than the final $\eta_{f}$. At this point the flow experiences an isothermal jump to the final state. Two highlighted points correspond to the isothermal jump condition. On scales smaller than the photon mean free path a weak fluid sub-shock results supersonic-subsonic transition than then smoothly evolves toward the final state.

\subsection{Appearance of the iso-thermal jump}

The above derivation assumed that the upstream medium is cold, so that the shock is infinitely strong. If the upstream plasma has temperature $\theta_{1}$ (so that Mach number is $M_{1}=\sqrt{\frac{\mu}{\gamma \theta_{1}}} \beta_{1}$ ), the compression ratio is

$$
\eta_{ \pm}=\frac{1}{2}\left(\frac{\theta_{1}}{\beta_{1}^{2} \mu}-\sqrt{\left(\frac{\theta_{1}}{\beta_{1}^{2} \mu}+1\right) 2-\frac{4 T}{\beta_{1}^{2} \mu}}+1\right)
$$




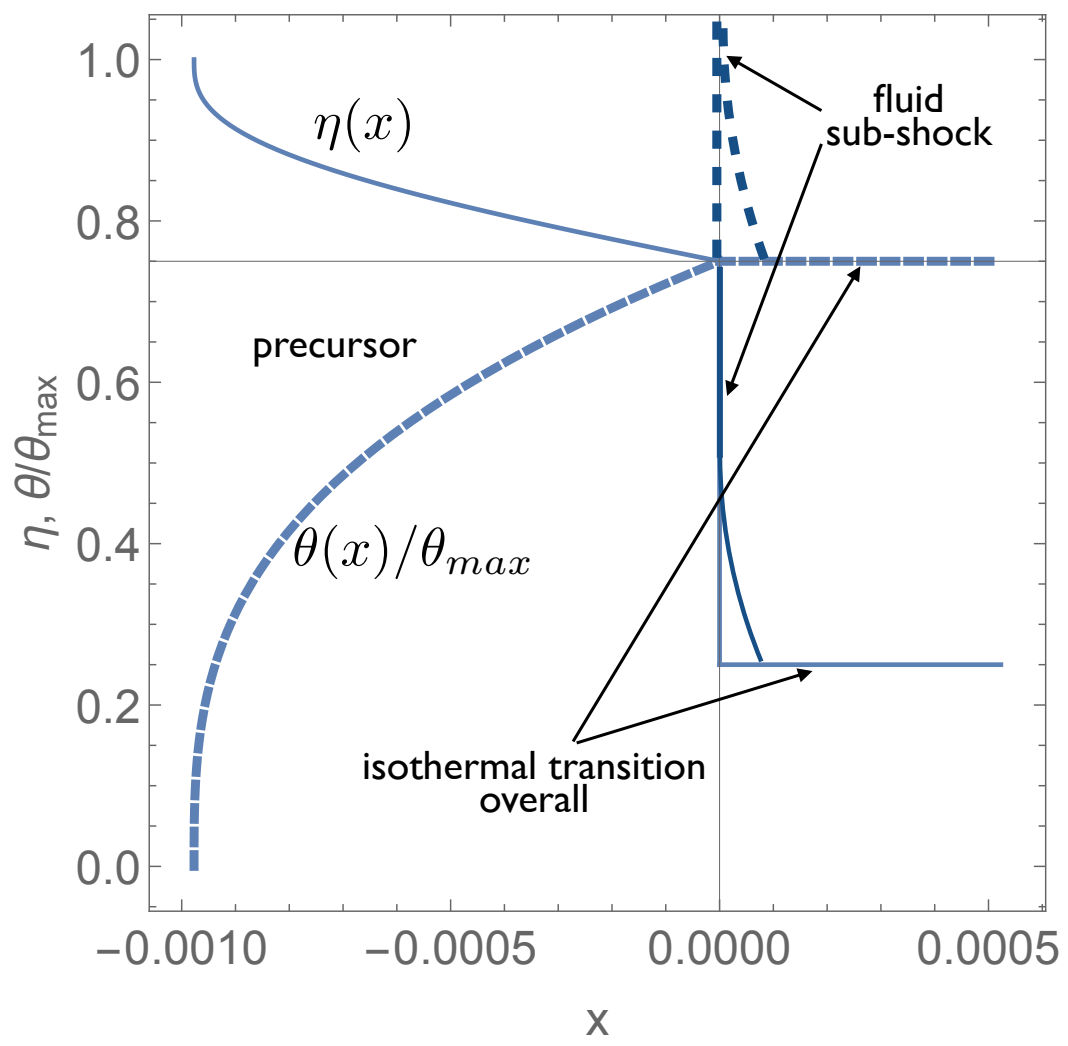

Fig. 3.- Compression ratio and temperature evolution within an isothermal transition, analytical solution of Eq. (36). The precursor extends to a finite distance in front of the isothermal transition (for cold upstream); distance is normalized to $L_{p}$, Eq. (35). In the precursor the flow is heated and decelerated by the radiation pressure Near the front of the precursor located at $x=x_{p} \approx-0.00097$ the compression ratio is $\eta=1-(6(x-x-p))^{1 / 3}$. The isothermal jump is located at $x=0$, where the final temperature $\theta_{2}=(3 / 4) \theta_{\max }$ and compression $\eta=1 / 4$ are reached. On scales much smaller than the radiative diffusion length the isothermal jump consist of weak sub-shock and a narrow relation layer. For $\gamma=5 / 3$ the subshock heats the plasma to the maximal possible temperature of $\theta_{\max }=\mu \beta_{1}^{2} / 4$.

Thus, the maximal temperature is

$$
\theta_{\max }=\frac{\left(\beta_{1}^{2} \mu+\theta_{1}\right)^{2}}{4 \beta_{1}^{2} \mu}=\frac{\beta_{1}^{2} \mu\left(\gamma M_{1}^{2}+1\right)^{2}}{4 \gamma^{2} M_{1}^{4}}
$$


Post-shock temperature and compression ratios are

$$
\begin{aligned}
\theta_{2} & =-\frac{2(\gamma-1) \gamma \theta_{1}^{2}}{(\gamma+1)^{2} \beta_{1}^{2} \mu}+\frac{2(\gamma-1) \beta_{1}^{2} \mu}{(\gamma+1)^{2}}-\frac{\left(\gamma^{2}-6 \gamma+1\right) \theta_{1}}{(\gamma+1)^{2}} \\
\eta_{2} & =\frac{(\gamma-1) \beta_{1}^{2} \mu+2 \gamma \theta_{1}}{(\gamma+1) \beta_{1}^{2} \mu}
\end{aligned}
$$

Equating $\theta_{\max }$ to $\theta_{2}$ we find that isothermal jump forms for

$$
\theta_{1}<\frac{(3-\gamma) \beta_{1}^{2} \mu}{3 \gamma-1}, M_{1}>M_{c r i t}=\sqrt{\frac{3 \gamma-1}{(3-\gamma) \gamma}}=\frac{3}{\sqrt{5}}=1.34
$$

At this point $p_{2} / p_{1}=(\gamma+1) /(3-\gamma)$, cf. , Landau \& Lifshitz (1959) Eq. (95.7).

Note that the ratio of the final temperature $\theta_{2}$ to maximal temperature $\theta_{\max }$ never exceeds unity:

$$
\begin{aligned}
& \frac{\theta_{2}}{\theta_{\text {max }}}=\frac{-8(\gamma-3)^{2}(\gamma-1) \gamma+4\left(3 \gamma^{4}-28 \gamma^{3}+66 \gamma^{2}-28 \gamma+3\right) m_{1}^{2}+8(1-3 \gamma)^{2}(\gamma-1) m_{1}^{4}}{(\gamma+1)^{2}\left(-\gamma+(3 \gamma-1) m_{1}^{2}+3\right)^{2}} \leq 1 \\
& m_{1}=\frac{M_{1}}{M_{\text {crit }}}
\end{aligned}
$$

This ratio reaches unity only at $m_{1}=1$. In this case

$$
\eta_{\text {crit }}=\frac{1+\gamma}{3 \gamma-1}=2 / 3
$$

Overall properties of the isothermal jump for strong shocks are further discussed in Appendix B.

\subsection{Internal structure of the isothermal jump}

The appearance of the isothermal jump is a mathematically oddity, related to the diffusive approximation and the assumption of the local thermodynamics equilibrium. This prevent the formation of the temperature jump. Also, On the scales smaller than the photon mean free path the radiation becomes decoupled from the plasma. As a result a fluid subshock forms with a typical thickness much smaller than the photon mean free path.

Let us consider the properties of this fluid subshock. It will occur when the temperature of the flow reaches the final temperature $\theta_{2}$, while the local compression ratio is $\eta_{+}$. The corresponding Mach number (upstream of the fluid subshock) is

$$
M_{s, 1}=\frac{\eta_{+}}{\sqrt{\gamma \theta_{2}}}=\sqrt{\frac{2}{\gamma(\gamma-1)}}=\frac{3}{\sqrt{5}}=1.34
$$

(for $\gamma=5 / 3$, and only for this $\gamma, M_{s}$ equals $M_{c r i t}$, Eq. 40). The sub-shock is weak. 
A subshock with Mach number (43) has (the inverse) compression ratio $\eta_{s}$

$$
\eta_{s}=\gamma-1=2 / 3
$$

The corresponding temperature jump is

$$
\frac{\theta_{s}}{\theta_{2}}=(3-\gamma)=\frac{4}{3}
$$

while the post-shock temperature is

$$
\theta_{s, 2}=\frac{2(3-\gamma)(\gamma-1)}{(\gamma+1)^{2}} \mu \beta_{1}^{2}=\frac{1}{4} \mu \beta_{1}^{2}
$$

Thus, for $\gamma=5 / 3$ the subshock reaches the maximal possible temperature.

The total compression ratio from the upstream is

$$
\eta_{s, 2}=\eta_{+} \eta_{s u b}=2 \frac{\gamma-1}{\gamma+1}=1 / 2
$$

see Fig. 2

The post-fluid shock Mach number is

$$
M_{s, 2}=\sqrt{\frac{2(\gamma-1)}{(3-\gamma) \gamma}}=\sqrt{3 / 5}
$$

\section{Highly radiation-dominated shocks: no isothermal jump}

Above in $\S 4$ we considered the simplest most that demonstrates the formation of the isothermal jump. We neglected the contribution of radiation and pairs to mass and energy density (and pressure), but allowed for the diffusive energy redistribution within the flow by long range photon propagation.

Next, let us consider another extreme case of strongly radiation-dominated shocks, when pressure and energy density of matter can be neglected in comparison with radiation, yet when inertia is dominated by matter. Thus, we are in the regime

$$
\frac{\pi \theta^{4}}{15 \mu} \ll n \ll \frac{\pi \theta^{3}}{45}
$$

The flow is then described by the system of conservation laws

$$
\begin{aligned}
& \beta_{1} \rho_{1}=\beta \rho \\
& \rho_{1} \beta_{1}^{2}=p_{\text {rad }}+\rho \beta^{2} \\
& \rho_{1} \beta_{1}^{3} / 2=\left(w_{\text {rad }}+\rho \beta^{2} / 2\right) \beta+F_{r} \\
& w_{\text {rad }}=\frac{4}{3} u_{\text {rad }}=4 p_{\text {rad }} \\
& F_{r}=-\frac{4}{3} \frac{a c}{\left(\rho / m_{p}\right) \sigma_{T}} T^{3} \partial_{x} T
\end{aligned}
$$


We find

$$
\begin{aligned}
& \eta=1-\frac{6}{7}\left(\frac{\theta}{\theta_{\max }}\right)^{4} \\
& \frac{\mu \lambda_{C} \beta_{1}}{\alpha_{f}^{2}} \partial_{x} \theta=\frac{7 \pi}{135}\left(\frac{\left.1-\left(\theta / \theta_{\max }\right)^{4}\right)}{1-(6 / 7)\left(\theta / \theta_{\max }\right)^{4}}\right) \theta \theta_{\max }^{4} \\
& \theta_{\max }=\left(\frac{270}{7 \pi}\right)^{1 / 4} \mu^{1 / 4} n_{1}^{1 / 4} \beta_{1}^{1 / 2}
\end{aligned}
$$

The $\eta-\theta$ dependence in this case is strikingly different from the case where pressure is dominated by matter (but transfer by radiation), $\S 4$. Now, compressibility evolves smoothly with temperature until a final state

$$
\begin{aligned}
& \theta_{2}=\theta_{\max } \\
& \eta_{2}=1 / 7
\end{aligned}
$$

is reached. The typical scale of the shock is $\Delta x \sim \mu \lambda_{C} \beta_{1} / \alpha_{f}^{2}$ (transition layer becomes thicker with higher velocity.).

Equation (51) has an analytical solution (after dimensionalizing)

$$
x=\frac{135}{196 \pi} \theta_{\max }^{-4} \ln \left(\frac{\theta^{28}}{\theta_{\max }^{4}-\theta_{T}^{4}}\right)
$$

Fig. 4.

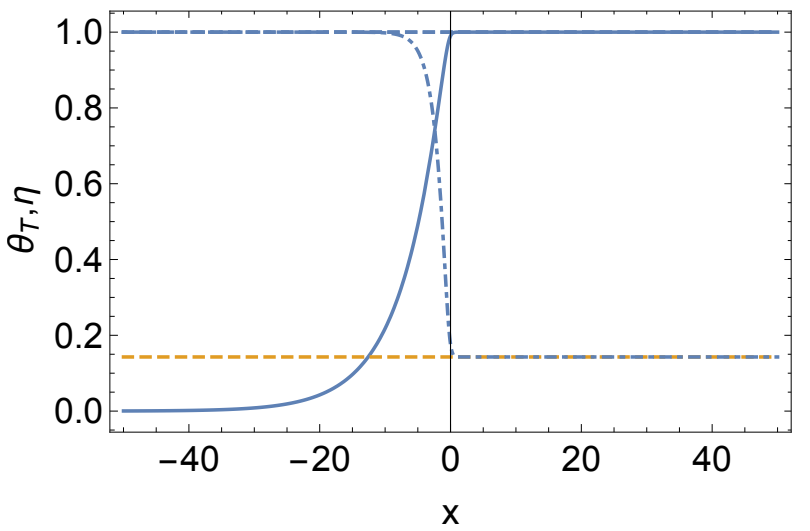

Fig. 4. - Structure of the radiation-dominated shock with no pair formation, scattering-dominated. For this plot $\theta_{\max }=1$. Solid line is $\theta$, dot-dashed line is $\eta$. Coordinate is normalized to $\mu \lambda_{C} \beta_{1} / \alpha_{f}^{2}$. The flow smoothly reaches the finite state without a discontinuous transitions.

Thus, for highly radiation-dominated shocks there is no isothermal jump. Mildly radiationdominated shocks do have isothermal jumps as we demonstrate next, $§ 5.1$ 


\subsection{Mildly radiation dominated shocks without pairs: reappearance of isothermal jump}

Let us keep radiation energy density and matter contribution to pressure. In this case illu-

minating analytical results can be obtained in the limit $\beta_{1}^{2} \rightarrow 0$ (but we keep $\beta_{1}^{2} \mu$ terms.) The momentum and energy conservation give

$$
\begin{aligned}
& \pi \eta \theta^{4}+45 n_{1}\left(\beta_{1}^{2}(\eta-1) \eta \mu+\theta\right)=0 \\
& \frac{\lambda_{C}}{\beta_{1} n_{1} \alpha_{f}^{2}} \partial_{x} \theta=\frac{8 \pi(\gamma-1) \eta \theta^{4}+45 n_{1}\left(\beta_{1}^{2}(\gamma-1)\left(\eta^{2}-1\right) \mu+2 \gamma \theta\right)}{3(\gamma-1) \eta \theta^{3}}
\end{aligned}
$$

Condition $\partial_{x} \theta=0$ corresponds to far downstream. In this case we can eliminate $n_{1}$ and find the relation between the final compression ratio and temperature (for a given $\beta_{1}$ ), Fig. 5

$$
\eta_{2}=\frac{1}{7}\left(4 \pm \sqrt{9+(56-42 \gamma) \frac{\theta_{2}}{\mu \beta_{1}^{2}(\gamma-1)}}\right)
$$

For $\theta_{2} \rightarrow 0$ this gives $\eta_{2}=1 / 7$ - compression ratio for strongly radiation-dominated shock (another root is the trivial $\eta_{2}=1$ ).

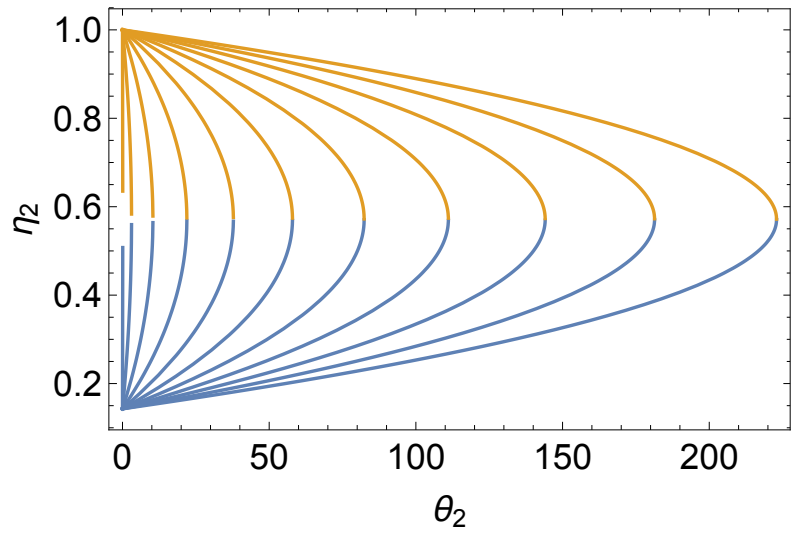

Fig. 5.- Dependence of $\eta_{2}\left(\theta_{2}\right)$ for different $\beta_{1}=0.01,0.06 \ldots 0.51$ in case of no pairs. Each curve corresponds to a given $\beta_{1}$; different points on the curve correspond to different $n_{1}$. Only lower parts of the curves correspond to shock transitions. For each $\theta_{2}<\theta_{\max }$ the final state is reached via vertical isothermal jump between points on the curves.

We can also solve for compression ratio as a function of upstream parameters, Fig. 6. Compression ratio never goes below $1 / 7$.

Finally, let us investigate how the final state can be reached. Here the situation is very similar to the simplest case considered in $\S 4$. As the flow evolves on the $\eta-\theta$ diagram from the initial point $\eta=1, \theta=0$, the final temperature is reached at smaller compression ratios. As a result, isothermal shock forms, Fig. 7. According to results in $\S 5$, for sufficiently small densities/high velocities the isothermal jump should disappear. This is indeed seen in Fig. 7, right panel. 

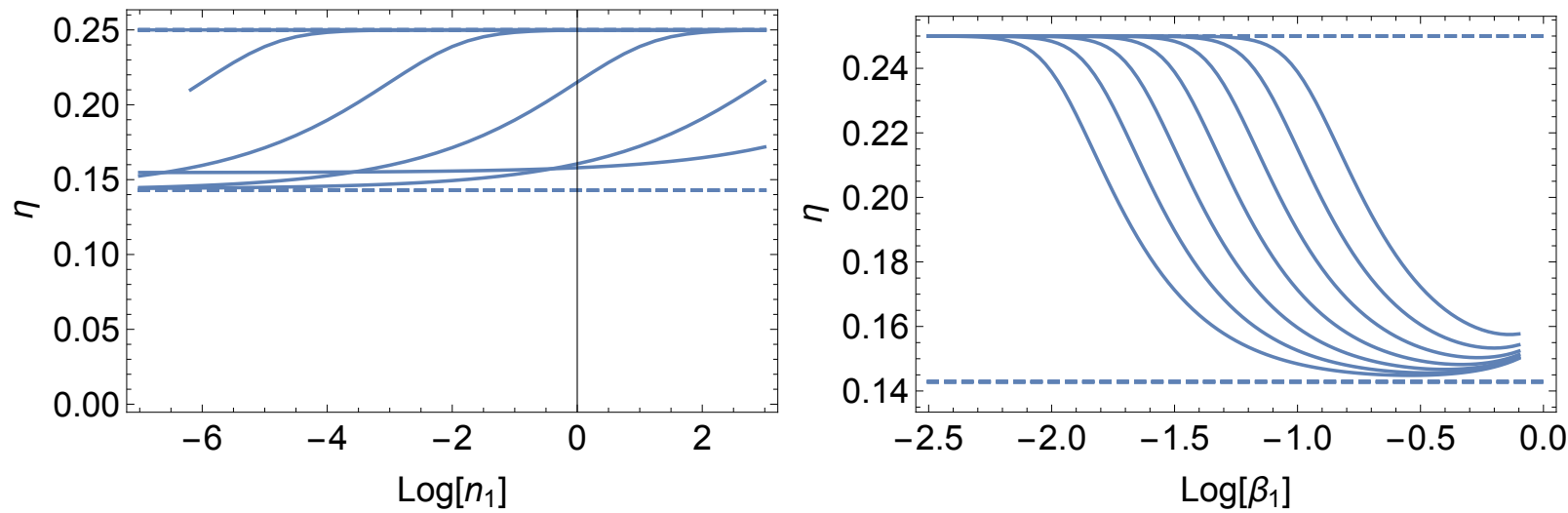

Fig. 6.- Compression ratio as function of shock velocity and upstream density; no pair creation. For high upstream density or low velocity the shock is matter-dominated with compression ratio approaching $1 / 4$, while for small density/high velocity the shock is radiation-dominated with compression ratio approaching $1 / 7$. The compression ratio never goes above 7 .
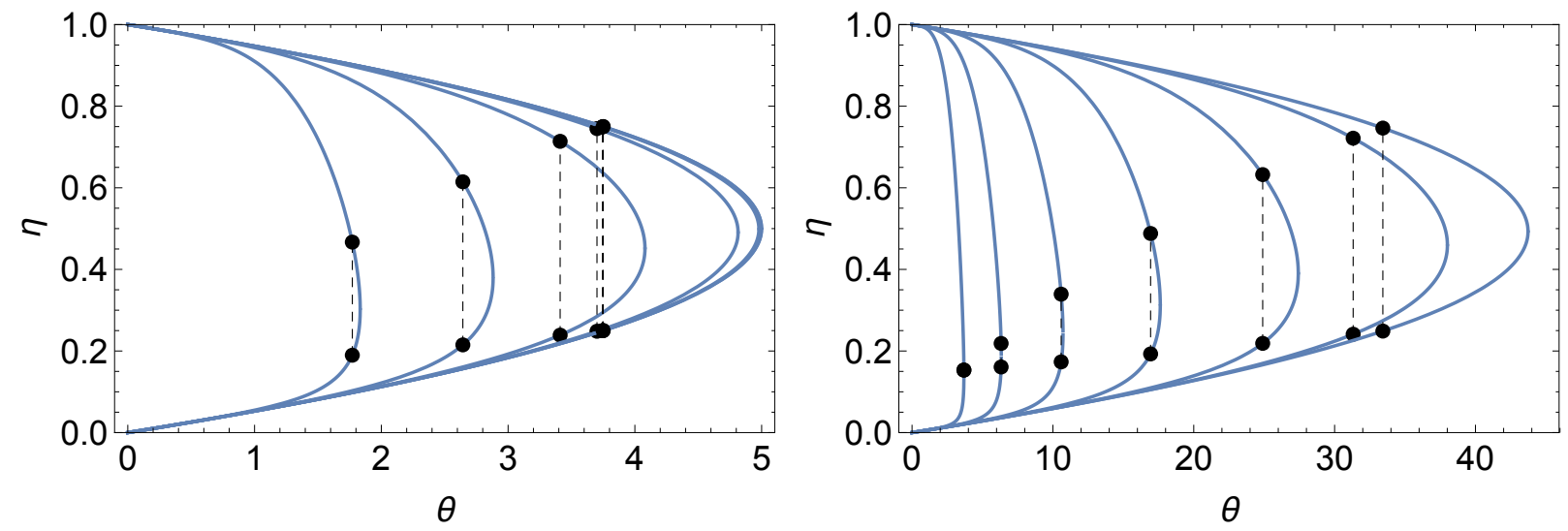

Fig. 7.- Evolution of flow without pair production on $\eta-\theta$ diagram. The flow evolves from the initial point $\eta=1, \theta=0$, the final temperature is reached at smaller compression ratios, upper dots. The isothermal jump forms, dashed lines. Different curves correspond to different densities, $n=10^{-1}, 1 \ldots 10^{5}$. Left panel: $\beta_{1}=0.1$, right panel $\beta_{1}=0.3$. For sufficiently small densities the isothermal jump disappears.

\subsection{The general case: radiation mediated shocks with pair production}

After getting experience with simple cases we are ready to tackle the full problem: flow with radiation and pair production We again make non-relativistic approximation, neglecting $\beta_{1}^{2} \ll 1$, 
but not when it comes with $\mu \gg 1$. The momentum and energy conservation equations give

$$
\begin{aligned}
& 45 \pi^{3 / 2} n_{1}\left(\theta-\beta_{1}^{2}(1-\eta) \eta \mu\right)+45 \sqrt{2} \eta \sqrt{g_{E}} e^{-1 / \theta} \theta^{5 / 2}+\pi^{5 / 2} \eta \theta^{4}=0 \\
& \frac{3 \pi^{3 / 2} \eta \theta^{3} \lambda_{C}}{\beta_{1} \alpha_{f}^{2}} \partial_{x} \theta=\left(\sqrt{2} \eta g_{e} e^{-1 / \theta} \theta^{3 / 2}+\pi^{3 / 2} n_{1}\right) \times \\
& \left(\frac{90 \sqrt{2} \eta g_{e} e^{-1 / \theta}(4 \theta+1) \theta^{3 / 2}}{\pi^{3 / 2}}+8 \pi \eta \theta^{4}+\frac{45 n_{1}\left(\beta_{1}^{2}(\gamma-1)\left(\eta^{2}-1\right) \mu+2 \gamma \theta\right)}{\gamma-1}\right)
\end{aligned}
$$

In the momentum equation terms are plasma contribution, pairs and radiation consequently.

Setting $\partial_{x} \theta=0$ in (57) determines the overall shock jump conditions. Since (57) is linear in $n_{1}$, we can eliminate $n_{1}$ and find how the compression ratio far downstream depends on the final temperature (for fixed $\beta_{1}$ ), Fig. 8

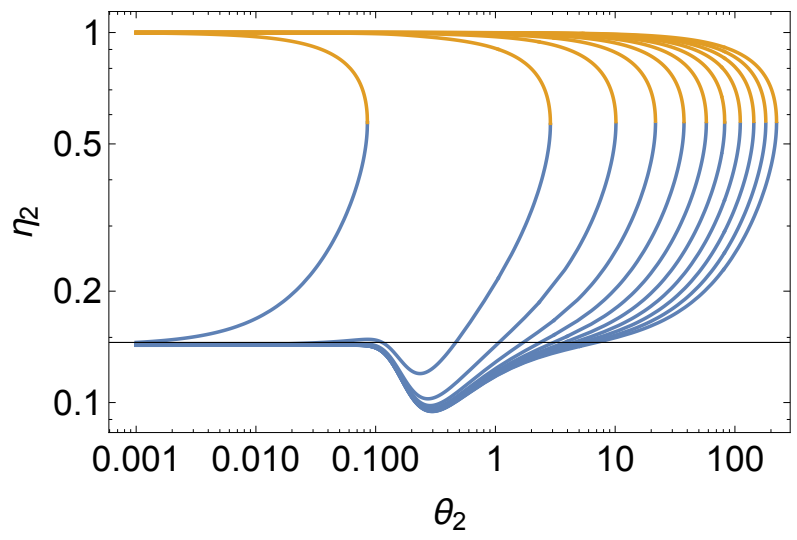

Fig. 8.- Dependence of $\eta_{2}\left(\theta_{2}\right)$ for different $\beta_{1}=0.01,0.06 \ldots 0.51$. Each curve corresponds to a given $\beta_{1}$; different points on the curve correspond to different $n_{1}$. Only lower parts of the curves correspond to shock transitions. For each $\theta_{2}<\theta_{\max }$ the final state is reached via vertical isothermal jump between points on the curves.

Few points are worth mentioning. At mild post-shock conditions the compression ratio can go below $1 / 7$ and reach $\sim 1 / 10$. This fairly mild modification is due to the formation of pairs - energy is spent on creating mass, not pressure. The effect is fairly mild since at smaller temperature there are few pairs, while at larger temperature pairs behave like radiation, so that their mass is not important. There is also a limiting case for large $n_{1}$, where pairs and radiation are not important, see $(29)$.

The shock jump conditions are plotted in Fig. 9, where the final compression ration $\eta_{2}$ and the final temperature $\theta_{2}$ are plotted as functions of the upstream density and shock velocity

Evolution of quantities within the shock in $\eta-\theta$ plane are pictured in Figs. 10 and 11. Each panel is for a given velocity $\beta_{1}$ with different curves corresponding to different densities. For sufficiently small $n_{1}$ the isothermal jump disappears. We know that when the post-shock pressure 

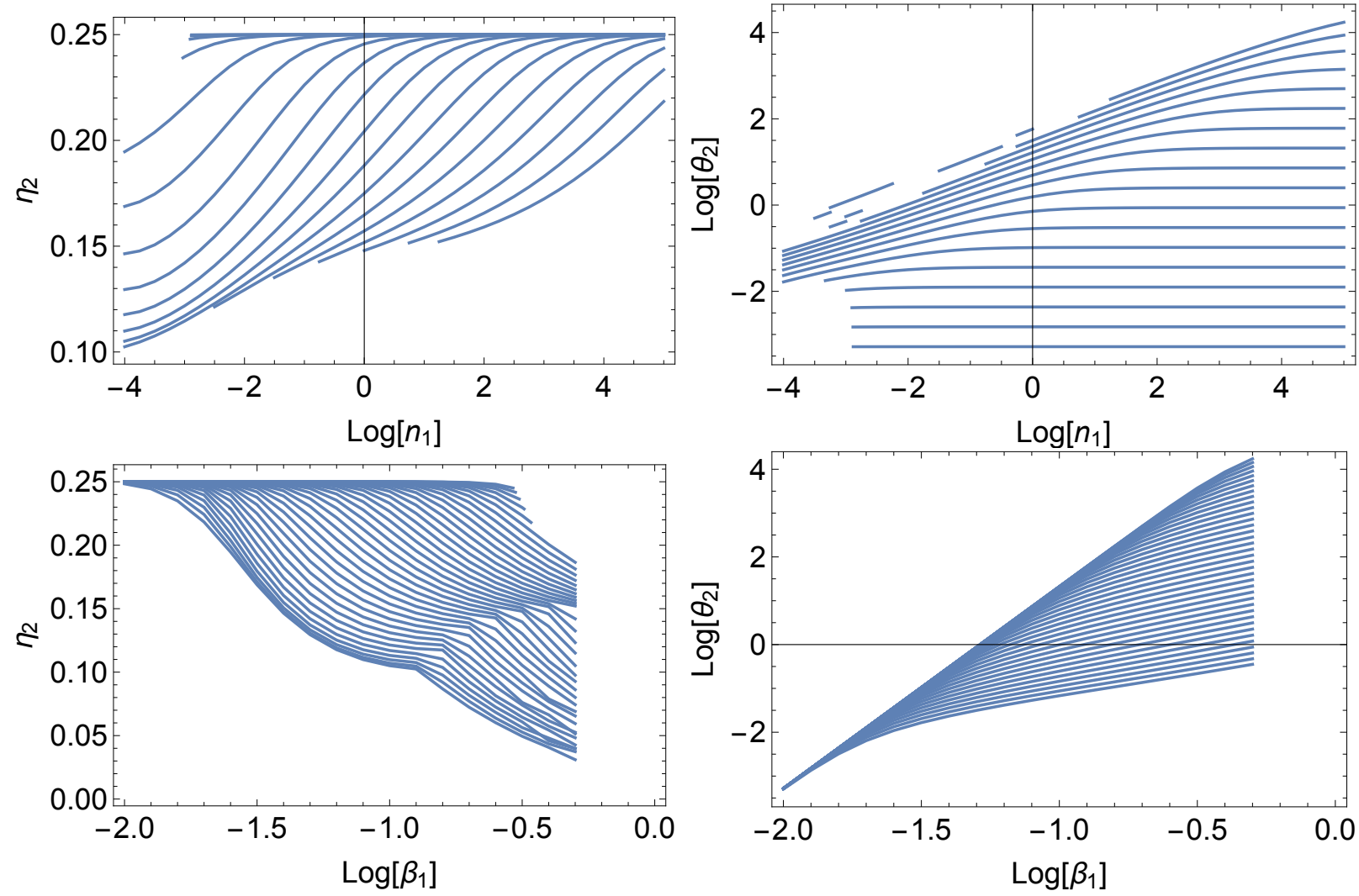

Fig. 9.- Jump conditions $\eta_{2}$ and $\theta_{2}$ for pair and radiative loaded shocks as function of upstream density for different shock velocities (top row, for $\beta_{1}=10^{-2}, 10^{-1.75}, \ldots, 10^{-0.25}$ ) and as function of shock velocity for different upstream density (bottom row, $n_{1}=10^{-4}, 10^{-3.75}, \ldots 10^{4}$ ).
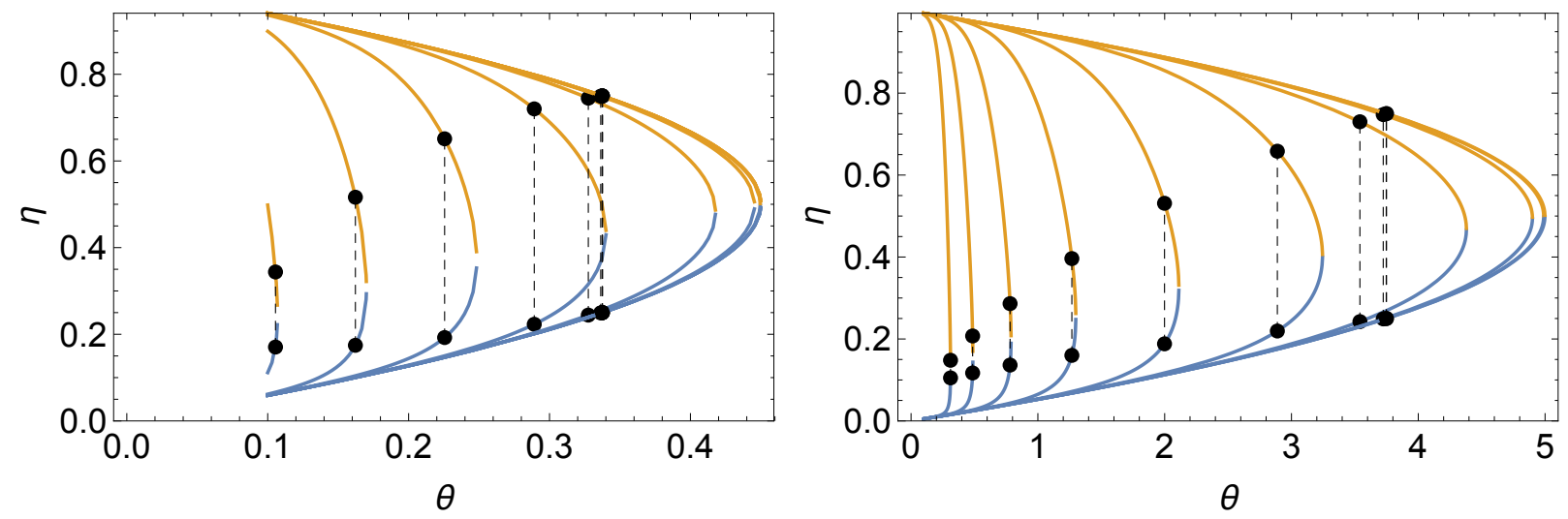

Fig. 10. - Evolution of pair and radiative loaded flows on $\eta-\theta$ plane for $\beta_{1}=0.03,0.1, n_{1}=$ $10^{-4}, 10^{-3} \ldots 10^{5}$.

and enthalpy are dominated by radiation, there is no isothermal jump - see $\S 5$. Analytically this can be seen from Eq. (56) where the only possibly negative term is proportional to density $n_{1}$. 


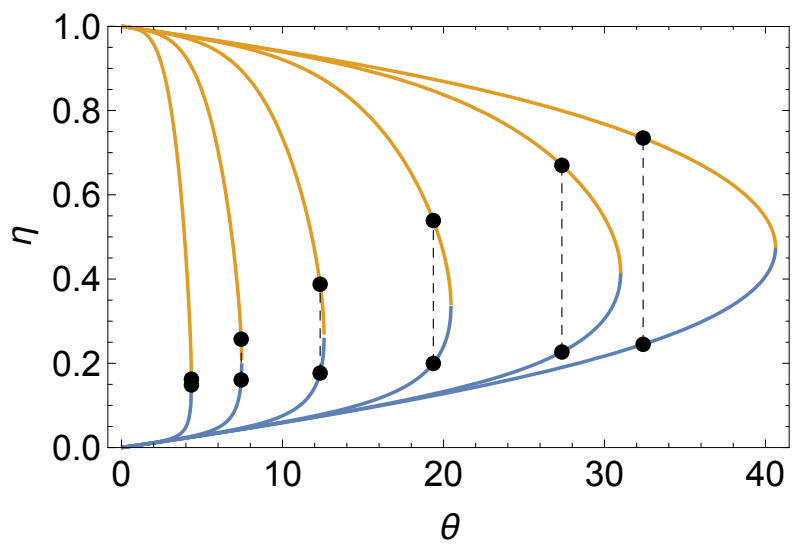

Fig. 11. - Evolution of pair and radiative loaded flows on $\eta-\theta$ plane for $\beta_{1}=0.3, n_{1}=1,10 \ldots 10^{5}$. For small densities the isothermal jump disappears.

Numerical solutions give the following conditions for the disappearance of the isothermal jump, Fig. 12.

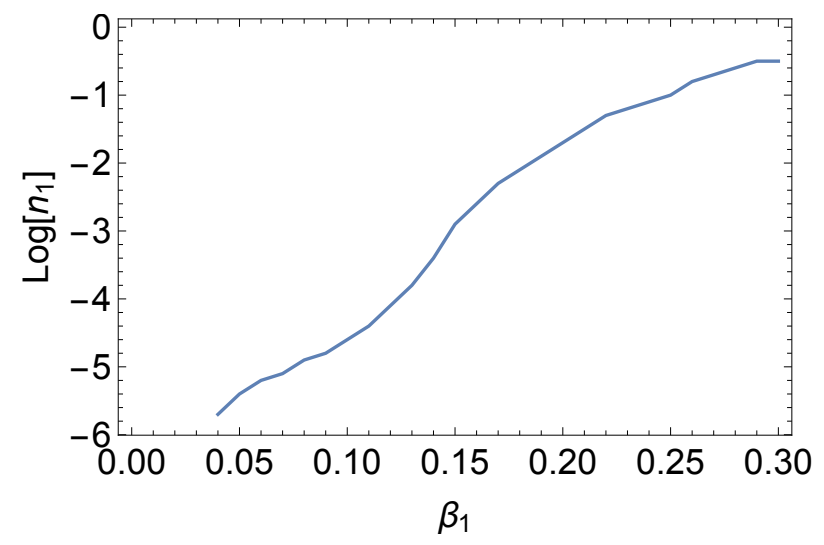

Fig. 12.- Condition for the appearance of the isothermal jump. For a given shock velocity the isothermal jump appears for densities above the curve.

\section{Limitations of the approach}

Our treatment has a number of simplifications/limitations. We treat photon propagation in diffusive approxmation, neglecting the spectral distribution and, eefectively using only the first two momenta of the photon distribution - photon density and flux. These are important limitations (see, e.g. Ito et al. 2018, for the kinetic treatment of photons). Yet this simplification allows to elucidate clearly the underlying effects. We expect that far upfront the diffusive approximation will be violated (see also Zeldovich \& Raizer 2003, for discussion of the applicability of the diffusive approximation). 
Another important limitation is the establishment of the black-body spectrum. Using the bremsstrahlung emissivity (e.g. Lang 1999) the time $\tau_{p h o t}$ to produce enough photons can be estimated as

$$
\tau_{p h o t} \approx \frac{\pi}{60} \frac{1}{\alpha_{f}^{3}} \frac{\lambda_{C}}{c} \frac{\theta^{7 / 2}}{n^{2}}
$$

(here $\alpha_{f}$ is the fine structure constant and $n$ is a dimensionless number density normalized to $\lambda_{C}^{3}$ ). This time is shorter than the scattering time $\tau_{\text {scat }}=1 /\left(n \sigma_{T} c\right)$ for

$$
\theta \leq \alpha_{f}^{2 / 7} n^{2 / 7} \approx 0.3 n^{2 / 7}
$$

(If number density of leptons is dominated by pairs, then $\tau_{\text {phot }}$ is always larger than $\tau_{\text {scat }}$, reaching a minimum at $\theta=1 / 2$, a which point $\tau_{\text {phot }} \approx 500 \tau_{\text {scat }}$.) The two-photon pair production can also be important (Budnik et al. 2010; Lundman et al. 2017), yet the long times to reach the blackbody photon numbers is a limitation of the approach.

Partly offsetting this problem is the fact that the Compton $y$-parameters on the scale of one optical depth, $y \sim 4 \theta$ is close to unity for $\theta \geq 0.1$. Also, the precursor is typically much larger than the mean free path, see Eq. (35). This implies that as the plasma is heated in the precursor, there is a lack of photons, but their typical energy is close to the equilibrium with the plasma temperature. As a result the typical photon energy density, and the corresponding effect on the energy redistribution, will be somewhat smaller.

\section{Discussion}

In this paper we address the structure of pair- and radiation-loaded shock transitions. The first most important effect is the energy redistribution within the flow due to diffusive propagation of radiation. This generically leads to the formation of a special surface within the flow - the isothermal jump. (The isothermal jump is different from highly radiative isothermal shocks; in our case the flow is energy conserving.) The isothermal jump forms first for plasma parameters when energy density of radiation and pair can be neglected, yet energy redistribution within the flow changes the flow properties qualitatively - even small energy density of radiation increases the order of the governing differential equation and thus cannot be neglected. The jump itself, on scales much smaller than the radiation scattering scale, consists of a mild shock and a narrow relation layer.

For increasing post-shock temperature the role of radiation pressure increases with respect to plasma pressure. As a result, for sufficiently strong shocks the isothermal jump disappears - highly radiation-dominated shocks smoothly reach the final state.

Overall, the formation of pairs has only a mild effect on the shock structure in the regime $\theta \sim 1$. This is due to the fact that at sub-relativistic temperatures the number of pairs is exponentially 
suppressed, while at highly relativistic regime thermodynamically pairs behave similar to photons. But formation of pairs may be important for the diffusive approximation to apply.

The parameters of radiation-modified shocks in the wind of the NS-NS mergers, see Eq. (3), are expected to be in a highly radiation-dominated regime (compare with Fig. 10), where the isothermal jump disappears. On the other hand, the isothermal jump could be important for core-

collapse SNe: the immediate post-shock temperature could be $25 \%$ higher than predicted by fluid jump conditions (but local density $30 \%$ lower). This could influence the neutrino flux, and, perhaps, help drive the explosion (see, e.g. Weaver 1976)

I would like to thank Maxim Barkov and Amir Levinson for discussions and organizers of the workshop "Cosmic Accelerators" at the Joint Space-Science Institute where part of this work has been performed. This work had been supported by NSF grant AST-1306672, DoE grant desc0016369 and NASA grant 80NSSC17K0757.

\section{REFERENCES}

Abbott, B. P., et al. 2017, ApJ, 848, L13

Bromberg, O., Tchekhovskoy, A., Gottlieb, O., Nakar, E., \& Piran, T. 2017, ArXiv e-prints

Budnik, R., Katz, B., Sagiv, A., \& Waxman, E. 2010, ApJ, 725, 63

Chapline, G. F., \& Granik, A. 1984, Physics of Fluids, 27, 1991

Gottlieb, O., Nakar, E., Piran, T., \& Hotokezaka, K. 2017, ArXiv e-prints

Ito, H., Levinson, A., Stern, B. E., \& Nagataki, S. 2018, MNRAS, 474, 2828

Landau, L. D., \& Lifshitz, E. M. 1959, Fluid mechanics

Lang, K. R. 1999, Astrophysical formulae

Lazzati, D., López-Cámara, D., Cantiello, M., Morsony, B. J., Perna, R., \& Workman, J. C. 2017, ApJ, 848, L6

Lundman, C., Beloborodov, A., \& Vurm, I. 2017, ArXiv e-prints

Metzger, B. D., et al. 2010, MNRAS, 406, 2650

Mezzacappa, A., Liebendörfer, M., Messer, O. E., Hix, W. R., Thielemann, F.-K., \& Bruenn, S. W. 2001, Physical Review Letters, 86, 1935

Pozanenko, A., et al. 2017, ArXiv:1710.05448

Rayleigh, L. 1910, Proceedings of the Royal Society of London Series A, 84, 247 
Svensson, R. 1984, MNRAS, 209, 175

Wandel, A., \& Yahil, A. 1979, A\&A, 72, 367

Weaver, T. A. 1976, ApJS, 32, 233

Zeldovich, Y. B., \& Raizer , Y. P. 2003, Physics of Shock Waves (Dover Publications Inc.)

\section{A. Finite Mach number: resolving the precursor}

Equation (31) has a special point upfront of the shock, at $\eta=1$, where the derivative $\partial_{x} \eta$ diverges, implying that the very front of the shock is located at finite distance with non-analytical behavior. Finite upstream Mach number resolves this singularity, as we demonstrate next.

For finite upstream temperature

$$
\begin{aligned}
& \rho_{1} v_{1}=\rho v \\
& p_{1}+\rho_{1} v_{1}^{2}=p+\rho v^{2} \\
& v_{1}\left(\frac{1}{2} \rho_{1} v_{1}^{2}+w_{1}\right)=F_{r}+v\left(\frac{\rho v^{2}}{2}+w\right) \\
& F_{r}=-\frac{4 \pi^{2} c^{3} m_{e} \theta_{T}^{3}}{45 n \lambda_{C}^{3} \sigma_{T}} \partial_{x} \theta_{T}
\end{aligned}
$$

Mass and momentum conservation give

$$
\begin{aligned}
& \eta=\frac{ \pm \sqrt{\left(\theta_{T, 1}+\beta_{1}^{2} \mu\right)^{2}-4 \beta_{1}^{2} \mu \theta_{T}}+\theta_{T, 1}+\beta_{1}^{2} \mu}{2 \beta_{1}^{2} \mu} \rightarrow \frac{1}{2} \pm \frac{\sqrt{\beta_{1}^{2} \mu-4 \theta_{T}}}{2 \beta_{1} \sqrt{\mu}} \\
& \frac{1}{2}\left(1-\sqrt{1-\frac{\theta_{T}}{\theta_{T, \max }}}\right) \\
& \theta_{T}=\eta\left(\theta_{T, 1}-\beta_{1}^{2}(\eta-1) \mu\right) \rightarrow \beta_{1}^{2}(1-\eta) \eta \mu
\end{aligned}
$$

where the second relations indicate $\theta_{T, 1} \rightarrow 0$ limit.

Thus, there are two branches for $\eta(\theta)$ which connect at

$$
\begin{aligned}
& \eta_{\text {crit }}=\frac{1}{2}+\frac{\theta_{T, 1}}{2 \beta_{1}^{2} \mu} \rightarrow \frac{1}{2} \\
& \theta_{T, \max }=\frac{\left(\theta_{T, 1}+\beta_{1}^{2} \mu\right)^{2}}{4 \beta_{1}^{2} \mu} \rightarrow \frac{\beta_{1}^{2} \mu}{4}
\end{aligned}
$$


The equation for the compression ratio becomes

$$
\frac{\pi \beta_{1}^{5}(\gamma-1) \mu^{3}}{15 \alpha_{f}^{2} n_{1}^{2} \lambda_{C}^{5}} \partial_{x} \eta=\frac{\beta_{1}^{6}(\eta-1) \mu^{3}\left(-2 \gamma \theta_{T, 1}+\beta_{1}^{2} \gamma(\eta-1) \mu+\beta_{1}^{2}(\eta+1) \mu\right)}{\eta^{4}\left(\beta_{1}^{2}(\eta-1) \mu-\theta_{T, 1}\right)^{3}\left(\theta_{T, 1}+\beta_{1}^{2}(1-2 \eta) \mu\right)} \rightarrow \frac{\gamma(\eta-1)+\eta+1}{(1-2 \eta)(1-\eta)^{2} \eta^{4}}
$$

For finite $\theta_{T, 1}$ the compression ratio far upstream,

$$
\eta \propto e^{\frac{30 \beta_{1} n_{1}^{2} z \lambda_{C}^{5} \alpha_{f}^{2}}{\pi(\gamma-1) \theta_{T, 1}^{3}}}
$$

is finite for all $x$ (upstream medium corresponds to $x<0$ ). For small upstream temperature the bulk of the transition is well described by the $\theta_{T, 1}=0$ limit.

\section{B. Properties of the isothermal jump}

Let us highlight the properties of the isothermal jump in the simple,analytically treatable case, when the radiation and pair production effects can be neglected, while energy redistribution by photon diffusion is important, see $\S 4$.

At the iso-thermal jump the sound speed is

$$
c_{s}=\sqrt{\gamma T / m_{p}}=\frac{\sqrt{2} \sqrt{\gamma-1} \sqrt{\gamma}}{\gamma+1} v_{1}
$$

At this point, on the upper branch the parameters of the flow are

$$
\begin{aligned}
& \eta_{+}=2 /(\gamma+1) \\
& v_{+}=\frac{2}{\gamma+1} v_{1}=\frac{3}{4} v_{1} \\
& M_{+}=\frac{\sqrt{2}}{\sqrt{\gamma-1} \sqrt{\gamma}}=\frac{3}{\sqrt{5}}
\end{aligned}
$$

While in the post-jump flow

$$
\begin{aligned}
& \eta_{2}=\frac{\gamma-1}{\gamma+1} \\
& v_{2}=\frac{\gamma+1}{\gamma-1} v_{1}=\frac{1}{4} v_{1} \\
& M_{2}=\frac{\sqrt{\gamma-1}}{\sqrt{2 \gamma}}=\frac{1}{\sqrt{5}}
\end{aligned}
$$

Thus, the compression ratio at the isothermal jump is $(\gamma-1) / 2=1 / 3$. 


\title{
Radiation- and pair-loaded shocks
}

\author{
Maxim Lyutikov \\ Department of Physics and Astronomy, Purdue University, \\ 525 Northwestern Avenue, West Lafayette, IN 47907-2036
}

\begin{abstract}
We consider structure of mildly relativistic shocks in dense media, taking into account radiation and pair loading, and diffusive radiation transfer. For increasing shock velocity (increasing post-shock temperature), the first effect is the efficient energy redistribution by radiation within the shock that leads to the appearance of an isothermal jump, whereby the flow reaches the finial state through a discontinuous isothermal transition (which is not a shock). For sufficiently high temperatures the highly radiationdominated shocks do not form isothermal jump. Pair production can mildly increase the overall shock compression ratio to $\approx 10$ (from 4 for matter-dominated shocks and 7 of the radiation-dominated shocks).
\end{abstract}

\section{Introduction}

In many astrophysical settings shocks propagate through dense environment with mildly relativistic velocities, so that the post-shock radiation pressure can exceed the thermal pressure (Budnik et al. 2010). In addition, astrophysical shocks may heat up the plasma to temperatures where postshock pair thermal production may become important. For example, the discovery of GRB170817A associated with gravitational waves event GW170817 (Abbott et al. 2017) is best explained due to the emission from the jet propagating with mildly relativistic velocities through a dense wind generated by the accretion torus (Lazzati et al. 2017; Pozanenko et al. 2017; Gottlieb et al. 2017; Bromberg et al. 2017). In this paper we address the structure of pair and radiation loaded shock transitions.

Several non-standard, so to say, phenomena are expected in these types of shocks. First, some energy in the downstream medium will be converted into radiation and pairs, thus modifying the thermodynamic properties of plasma by both contributing to effective mass density and to pressure. Secondly, energy redistribution within the shock may have profound influence on the shock properties (Landau \& Lifshitz 1959; Zeldovich \& Raizer 2003). Especially important is photon diffusion - even if the energy density in photons is below the thermal energy density, the high photon mean free path may modify the shock considerably.

We assume that plasma is sufficiently dense so that radiation is trapped within the flow. The first most important effect is the diffusion of radiation - even if energy density and mass loading due to radiation (and of pairs) are small comparable to matter energy density, the redistribution of 
energy within the shocked flow is important. This leads to the formation of isothermal jump, Landau \& Lifshitz (1959); Zeldovich \& Raizer (2003) and $\S 3$. (To clarify the notation, the isothermal jump is different from highly radiatively cooled isothermal shocks; in our case the flow is energy conserving.) On the other hand, for very strong highly radiation-dominated shocks the pressure created by photons and pairs may dominate over the ion pressure. This introduces further complications: the isothermal jump can disappear, Zeldovich \& Raizer (2003) and §4.

\section{Protons, radiation and pairs}

Consider three contributions to the total enthalpy of plasma - from thermal motion of ions $w_{p}$, radiation $w_{r}$ and pairs $w_{ \pm}$(defined per unit volume). For radiation and plasma ions, the enthalpy can be written as

$$
\begin{aligned}
\frac{\lambda_{C}^{3}}{c^{2} m_{e}} w_{r} & =\frac{4 \pi \theta^{4}}{45} \\
\frac{\lambda_{C}^{3}}{c^{2} m_{e}} w_{p} & =\frac{\gamma \theta n}{\gamma-1}
\end{aligned}
$$

with $\gamma=5 / 3$ for the non-relativistic ion component. To dimensionalize the equations, we scaled temperature $\theta=T /\left(m_{e} c^{2}\right)$, and number density $n=n / \lambda_{C}^{3} ; \mu=m_{p} / m_{e}$.

The pair pressure, enthalpy and number density is a fairly complicated function of temperature, especially in a weakly relativistic regime (e.g. Wandel \& Yahil 1979; Svensson 1984, and reference there in). To simplify the consideration, we adopt the following parametrization

$$
\begin{aligned}
& \frac{\lambda_{C}^{3}}{c^{2} m_{e}} w_{ \pm}=\frac{\sqrt{2} g_{E} e^{-1 / \theta} \sqrt{\theta^{3}}(4 \theta+1)}{\pi^{3 / 2}} \\
& g_{E}=1+0.47 \theta+0.37 \sqrt{\theta}+\sqrt{\frac{2}{\pi}} \theta^{3 / 2} \zeta(3),
\end{aligned}
$$

see Wandel \& Yahil (1979); Svensson (1984), $\zeta$ is the zeta-function (thus, pairs are assumed to be relativistic, factor of $4 \theta$ above). We expect that these simplifications for the pairs equation of state introduce only mild corrections.

Thus, for plasma with ion number density $n$ (normalized to $\lambda_{C}^{-3}$ ) the radiation pressure dominates the ion pressure at

$$
n \leq \frac{\pi}{15} \theta^{3}
$$

while the radiation mass-density dominates the ion density at much lower $n$,

$$
n \leq \frac{\pi}{15} \frac{\theta^{3}}{\mu}
$$

For pairs, at sub-relativistic temperatures the pair mass density and pressure are exponentially suppressed, $\propto e^{-1 / \theta}$, while for $\theta \geq 1$ the pair contribution becomes comparable to that of radiation. 
For example, for $\theta=1$ the radiation mass density dominates ion density for $n<\pi /(15 \mu)=10^{-4}$ $\left(\rho<3 \times 10^{3} \mathrm{~g} \mathrm{~cm}^{-3}\right)$.

The relations discussed above can be adopted to shock-heated plasma. In this case for matterdominated regime

$$
\theta \approx 2 \frac{\gamma-1}{(1+\gamma)^{2}} \mu \beta_{1}^{2}=\frac{3}{16} \mu \beta_{1}^{2}
$$

If the upstream plasma has density $n_{1}$, the post-shock ion pressure become smaller than radiation pressure at

$$
\frac{2 \mu n_{1} \beta_{1}^{2}}{\gamma+1}<\frac{\pi}{45} \theta^{4} \rightarrow n<\frac{8 \pi(\gamma-1)^{4}}{45(\gamma+1)^{7}} \beta_{1}^{6} \mu^{3}
$$

In such highly radiation-dominated shock without pairs (still, mass density is dominated by ions)

$$
\begin{aligned}
& \eta=1 / 7 \\
& \theta_{\max }=\left(\frac{270}{7 \pi}\right)^{1 / 4} n_{1}^{1 / 4} \mu^{1 / 4} \beta_{1}^{1 / 2}
\end{aligned}
$$

Also, in the high compressibility limit $\eta \rightarrow 0$

$$
\theta_{T}=\left(\frac{45}{\pi}\right)^{1 / 4} \mu^{1 / 4} \beta_{1}^{1 / 2}
$$

which is very close to $(7)$.

For relativistic temperatures the condition (6) is also approximately the condition for pair pressure to dominate over ion kinetic pressure, while at $\theta \leq 1$ the post-shock ion pressure become smaller than pair pressure for

$$
n_{1} \leq \frac{4 \beta_{1}^{3}(\gamma-1)^{5 / 2} \mu^{3 / 2} e^{-\frac{(\gamma+1)^{2}}{2 \beta_{1}^{2}(\gamma-1) \mu}}}{\pi^{3 / 2}(\gamma+1)^{4}}
$$

Due to exponential dependence this ratio varies over large values at mild shock velocities.

\section{Radiation-mediated shocks}

\subsection{The first effect: formation of isothermal jump in strong shocks}

As the velocity of the shock grows, the energy redistribution within the flow by radiation becomes the first important effect - even if the radiation pressure is much smaller than the matter pressure. Let's neglect radiation energy density and pressure, but keep energy diffusion due to photons with large mean free path ( $c f$. Zeldovich \& Raizer 2003). Everywhere along the stationary 
flow we have conservation of matter, momentum and energy flux:

$$
\begin{aligned}
& \beta_{1} \rho_{1}=\beta \rho \\
& \rho_{1} \beta_{1}^{2}=\frac{\rho}{m_{p}} T+\rho \beta^{2} \\
& \rho_{1} \beta_{1}^{3} / 2=\left(\frac{\gamma}{\gamma-1} \frac{\rho}{m_{p}} T+\rho \beta^{2} / 2\right) \beta+F_{r} \\
& F_{r}=-\frac{c}{3 n \sigma_{T}} \nabla u_{r a d} \\
& u_{r a d}=\frac{4}{c} \sigma_{S B} T^{4}
\end{aligned}
$$

where subscript 1 indicates quantities far upstream. In (10) $F_{r}$ is the diffusive energy flux carried by radiation in the high optical depth limit.

Introducing the inverse of the compression ratio $\eta=n_{1} / n$ Eq. (10) becomes

$$
\begin{aligned}
& \theta=\beta_{1}^{2}(1-\eta) \eta \mu \\
& \frac{\lambda_{C}}{15 \beta_{1} n_{1}^{2} \alpha_{f}^{2}} \partial_{x} \theta=\frac{\beta_{1}^{2}\left(\eta^{2}-1\right) \mu+\frac{2 \gamma \theta}{\gamma-1}}{\eta \theta^{3}}
\end{aligned}
$$

Far downstream $\partial_{x} \theta=0$ and (11-12) give shock jump conditions

$$
\begin{aligned}
\eta_{2} & =\frac{\gamma-1}{\gamma+1} \\
\theta_{2} & =\frac{2 \beta_{1}^{2}(\gamma-1) \mu}{(\gamma+1)^{2}}
\end{aligned}
$$

Importantly, in order to reach this final state a flow should develop a special type of discontinuity - isothermal jump (Landau \& Lifshitz 1959; Zeldovich \& Raizer 2003). The isothermal jump in this case forms regardless of a particular form of $F_{r}$, as we discus next.

The momentum conservation (11) can also be written for the evolution of compression as a function of temperature $\eta(\theta)$

$$
\begin{aligned}
& \eta=\frac{1}{2}\left(1 \pm \sqrt{1-\frac{4 \theta}{\beta_{1}^{2} \mu}}\right)=\frac{1}{2}\left(1 \pm \sqrt{1-\frac{\theta}{\theta_{\max }}}\right) \\
& \theta_{\max }=\frac{\beta_{1}^{2} \mu}{4}
\end{aligned}
$$

Thus, there are two branches for $\eta(\theta)$; they connect at the point

$$
\begin{aligned}
\eta_{\text {crit }} & =\frac{1}{2} \\
\theta_{\max } & =\frac{\beta_{1}^{2} \mu}{4}
\end{aligned}
$$


Importantly, the initial point $\eta=1$ is on the upper branch, while the final jump conditions is on the lower branch. Yet the flow cannot pass continuously from the initial to the final point - using (11) the equation for the compression ratio becomes

$$
\frac{\pi \beta_{1}^{5}(\gamma-1) \mu^{3}}{15 \alpha_{f}^{2} \mathrm{n} 1^{2} \lambda_{C}^{5}} \partial_{x} \eta=\frac{\gamma(\eta-1)+\eta+1}{(1-2 \eta)(1-\eta)^{2} \eta^{4}}
$$

Equation (16) clearly shows that the final state of $\eta=\eta_{2}=1 / 4$ (for $\gamma=5 / 3$ ) cannot be reached continuously: in order to reach it continuously one must pass through a special point $\eta=1 / 2$. This statement is true for any non-zero left hand side.

Note, that

$$
\frac{\theta_{2}}{\theta_{\max }}=8 \frac{\gamma-1}{(\gamma+1)^{2}}=\frac{3}{4}<1
$$

Thus, as the state evolves along the upper branch, the terminal temperature is reached before the the terminal compression. It is required that temperature increase monotonically (e.g. Landau \& Lifshitz 1959, Eq. (95.3)). Thus, since $\theta_{2}<\theta_{\max }$, the final state cannot be reached continuously. There should be an isothermal jump at $\theta=\theta_{2}$, Fig. (1).

In fact we can find an exact analytical solution of (16). Let us normalize out,

$$
\partial_{x} \eta=\frac{\gamma(\eta-1)+\eta+1}{(1-2 \eta)(1-\eta)^{2} \eta^{4}}
$$

which has a formal solution for $x(\eta)$ (with $x(1)=0$ )

$$
\begin{aligned}
& x=-\frac{\left(\gamma^{2}+4 \gamma+11\right) \eta^{5}}{5(\gamma+1)^{3}}-\frac{4\left(\gamma^{2}-4 \gamma+3\right) \eta^{3}}{3(\gamma+1)^{5}}-\frac{2 \eta^{7}}{7(\gamma+1)}+\frac{(3 \gamma+7) \eta^{6}}{6(\gamma+1)^{2}}-\frac{(\gamma-3) \eta^{4}}{(\gamma+1)^{4}}- \\
& \left.\frac{2(\gamma-3)(\gamma-1)^{2} \eta^{2}}{(\gamma+1)^{6}}-\frac{4(\gamma-3)(\gamma-1)^{3} \eta}{(\gamma+1)^{7}}-\frac{4(\gamma-3)(\gamma-1)^{4} \log (\gamma(\eta-1)+\eta+1)}{(\gamma+1)^{8}}+\frac{813}{573440}-\frac{27 \log (2)}{65536}\right)
\end{aligned}
$$

Fig. 2 (temperature is related to compression ratio via (14) along the upper branch).

Near $x \approx 0$ the compression ratio and temperature is $\eta \propto x^{1 / 3}$ (so that $d \eta / d x$ diverges). This is a drawback of the neglect of the upstream temperature; finite shock Mach number leads to the appearance of an exponential precursor, Appendix A.

\subsection{Appearance of the iso-thermal jump}

The above derivation assumed that the upstream medium is cold, so that the shock is infinitely strong. If the upstream plasma has temperature $\theta_{1}$ (so that Mach number is $M_{1}=\sqrt{\frac{\mu}{\gamma \theta_{1}}} \beta_{1}$ ), the compression ratio is

$$
\eta_{ \pm}=\frac{1}{2}\left(\frac{\theta_{1}}{\beta_{1}^{2} \mu}-\sqrt{\left(\frac{\theta_{1}}{\beta_{1}^{2} \mu}+1\right)^{2}-\frac{4 T}{\beta_{1}^{2} \mu}}+1\right)
$$




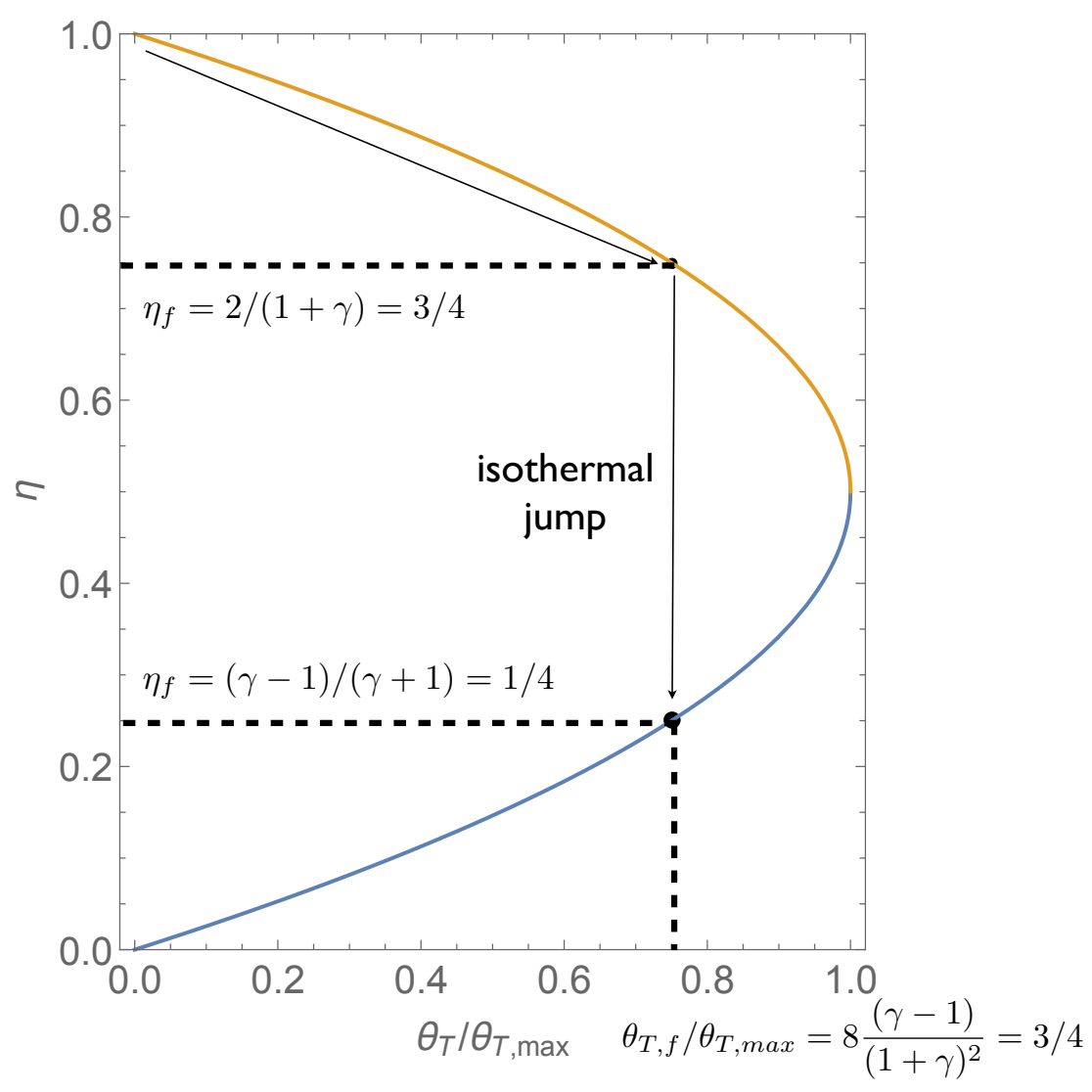

Fig. 1. - Evolution of the flow in the compression ratio - temperature plane. The flow starts at $\eta=1, \theta=0$, reaches finite temperature $\theta_{2}$ at smaller compression, experiences an isothermal jump to the final state. Two highlighted points correspond to the isothermal jump condition.

Thus, the maximal temperature is

$$
\theta_{\max }=\frac{\left(\beta_{1}^{2} \mu+\theta_{1}\right)^{2}}{4 \beta_{1}^{2} \mu}=\frac{\beta_{1}^{2} \mu\left(\gamma M_{1}^{2}+1\right)^{2}}{4 \gamma^{2} M_{1}^{4}}
$$

Post-shock temperature and compression ratios are

$$
\begin{aligned}
\theta_{2} & =-\frac{2(\gamma-1) \gamma \theta_{1}^{2}}{(\gamma+1)^{2} \beta_{1}^{2} \mu}+\frac{2(\gamma-1) \beta_{1}^{2} \mu}{(\gamma+1)^{2}}-\frac{\left(\gamma^{2}-6 \gamma+1\right) \theta_{1}}{(\gamma+1)^{2}} \\
\eta_{2} & =\frac{(\gamma-1) \beta_{1}^{2} \mu+2 \gamma \theta_{1}}{(\gamma+1) \beta_{1}^{2} \mu}
\end{aligned}
$$

Equating $\theta_{\max }$ to $\theta_{2}$ we find that isothermal jump forms for

$$
\theta_{1}<\frac{(3-\gamma) \beta_{1}^{2} \mu}{3 \gamma-1}, M_{1}>M_{\text {crit }}=\sqrt{\frac{3 \gamma-1}{(3-\gamma) \gamma}}=\frac{3}{\sqrt{5}}=1.34
$$



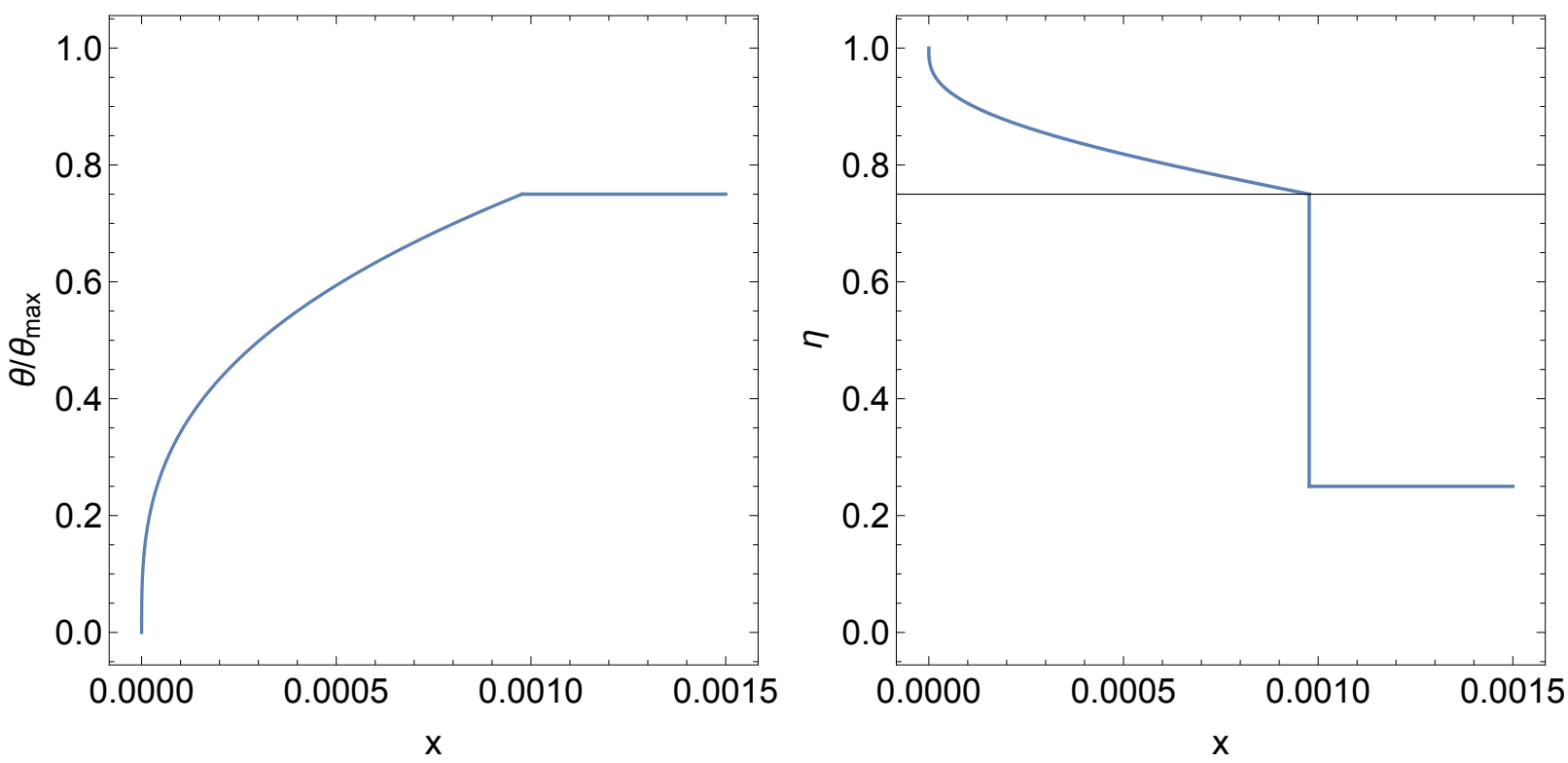

Fig. 2. - Compression ratio and temperature evolution within a simple radiation-mediated shock (19). The front of the shock transition is located at $x=0$. Near the front $\eta=1-(6 x)^{1 / 3}$. At finite distance there is an isothermal jump where the final temperature $\theta_{2}=(3 / 4) \theta_{\text {max }}$ and compression $\eta=1 / 4$ are reached.

At this point $p_{2} / p_{1}=(\gamma+1) /(3-\gamma)$, cf. , Landau \& Lifshitz (1959) Eq. (95.7).

Note that the ratio of the final temperature $\theta_{2}$ to maximal temperature $\theta_{\max }$ never exceeds unity:

$$
\begin{aligned}
& \frac{\theta_{2}}{\theta_{\text {max }}}=\frac{-8(\gamma-3)^{2}(\gamma-1) \gamma+4\left(3 \gamma^{4}-28 \gamma^{3}+66 \gamma^{2}-28 \gamma+3\right) m_{1}^{2}+8(1-3 \gamma)^{2}(\gamma-1) m_{1}^{4}}{(\gamma+1)^{2}\left(-\gamma+(3 \gamma-1) m_{1}^{2}+3\right)^{2}} \leq 1 \\
& m_{1}=\frac{M_{1}}{M_{\text {crit }}}
\end{aligned}
$$

This ratio reaches unity only at $m_{1}=1$. In this case

$$
\eta_{\text {crit }}=\frac{1+\gamma}{3 \gamma-1}=2 / 3
$$

Properties of the isothermal jump for strong shocks are further discussed in Appendix B.

\section{Radiation-dominated shocks without pair production}

\subsection{Highly radiation-dominated shocks: no isothermal jump}

Let us consider strongly radiation-dominated shocks, when pressure and energy density of matter can be neglected in comparison with radiation, yet when inertia is dominated by matter. 
Thus, we are in the regime

$$
\frac{\pi \theta^{4}}{15 \mu} \ll n \ll \frac{\pi \theta^{3}}{45}
$$

The flow is then described by the system of conservation laws

$$
\begin{aligned}
& \beta_{1} \rho_{1}=\beta \rho \\
& \rho_{1} \beta_{1}^{2}=p_{\text {rad }}+\rho \beta^{2} \\
& \rho_{1} \beta_{1}^{3} / 2=\left(w_{\text {rad }}+\rho \beta^{2} / 2\right) \beta+F_{r} \\
& w_{\text {rad }}=\frac{4}{3} u_{r a d}=4 p_{\text {rad }} \\
& F_{r}=-\frac{4}{3} \frac{a c}{\left(\rho / m_{p}\right) \sigma_{T}} T^{3} \partial_{x} T
\end{aligned}
$$

can be written as

$$
\begin{aligned}
& \eta=1-\frac{6}{7}\left(\frac{\theta}{\theta_{\max }}\right)^{4} \\
& \frac{\mu \lambda_{C} \beta_{1}}{\alpha_{f}^{2}} \partial_{x} \theta=\frac{7 \pi}{135}\left(\frac{\left.1-\left(\theta / \theta_{\max }\right)^{4}\right)}{1-(6 / 7)\left(\theta / \theta_{\max }\right)^{4}}\right) \theta \theta_{\max }^{4} \\
& \theta_{\max }=\left(\frac{270}{7 \pi}\right)^{1 / 4} \mu^{1 / 4} n_{1}^{1 / 4} \beta_{1}^{1 / 2}
\end{aligned}
$$

The $\eta-\theta$ dependence in this case is strikingly different from the case where pressure is dominated by matter (but transfer by radiation), $\S 3$. Now, compressibility evolves smoothly with temperature until a final state

$$
\begin{aligned}
\theta_{2} & =\theta_{\max } \\
\eta_{2} & =1 / 7
\end{aligned}
$$

is reached. The typical scale of the shock is $\Delta x \sim \mu \lambda_{C} \beta_{1} / \alpha_{f}^{2}$ (transition layer becomes thicker with higher velocity.).

Equation (28) has an analytical solution (after dimensionalizing)

$$
x=\frac{135}{196 \pi} \theta_{\text {max }}^{-4} \ln \left(\frac{\theta^{28}}{\theta_{\max }^{4}-\theta_{T}^{4}}\right)
$$

Fig. 3.

Thus, for highly radiation-dominated shocks there is no isothermal jump. Mildly radiationdominated shocks do have isothermal jumps as we demonstrate next, $§ 4.2$ 


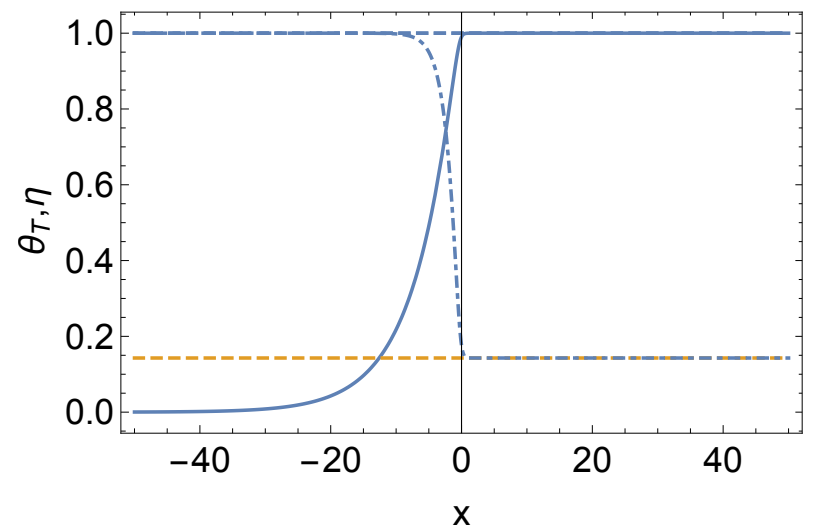

Fig. 3. - Structure of the radiation-dominated shock with no pair formation, scattering-dominated. For this plot $\theta_{\max }=1$. Solid line is $\theta$, dot-dashed line is $\eta$. Coordinate is normalized to $\mu \lambda_{C} \beta_{1} / \alpha_{f}^{2}$.

\subsection{Mildly radiation dominated shocks without pairs: reappearance of isothermal jump}

Let us keep radiation energy density and matter contribution to pressure. In this case illuminating analytical results can be obtained in the limit $\beta_{1}^{2} \rightarrow 0$ (but we keep $\beta_{1}^{2} \mu$ terms.) The momentum and energy conservation give

$$
\begin{aligned}
& \pi \eta \theta^{4}+45 n_{1}\left(\beta_{1}^{2}(\eta-1) \eta \mu+\theta\right)=0 \\
& \frac{\lambda_{C}}{\beta_{1} n_{1} \alpha_{f}^{2}} \partial_{x} \theta=\frac{8 \pi(\gamma-1) \eta \theta^{4}+45 n_{1}\left(\beta_{1}^{2}(\gamma-1)\left(\eta^{2}-1\right) \mu+2 \gamma \theta\right)}{3(\gamma-1) \eta \theta^{3}}
\end{aligned}
$$

Condition $\partial_{x} \theta=0$ corresponds to far downstream. In this case we can eliminate $n_{1}$ and find the relation between the final compression ratio and temperature (for a given $\beta_{1}$ ), Fig. 6

$$
\eta_{2}=\frac{1}{7}\left(4 \pm \sqrt{9+(56-42 \gamma) \frac{\theta_{2}}{\mu \beta_{1}^{2}(\gamma-1)}}\right)
$$

For $\theta_{2} \rightarrow 0$ this gives $\eta_{2}=1 / 7$ - compression ratio for strongly radiation-dominated shock (another root is the trivial $\eta_{2}=1$ ).

We can also solve for compression ratio as a function of upstream parameters, Fig. 5. Compression ratio never goes below $1 / 7$.

Finally, let us investigate how the final state can be reached. Here the situation is very similar to the simplest case considered in $\S 3$. As the flow evolves on the $\eta-\theta$ diagram from the initial point $\eta=1, \theta=0$, the final temperature is reached at smaller compression ratios. As a result, isothermal shock forms, Fig. 6.

According to results in $\S 4.1$, for sufficiently small densities/high velocities the isothermal jump should disappear. This is indeed seen in Fig. 6, right panel. 


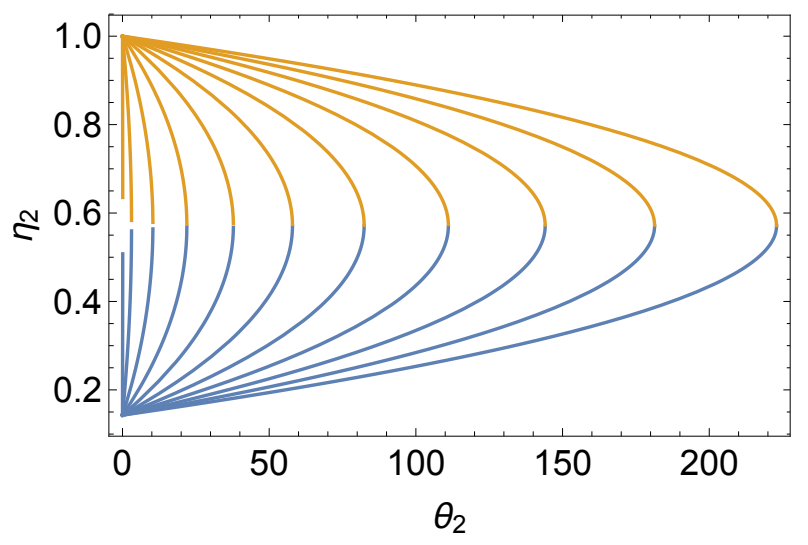

Fig. 4.- Dependence of $\eta_{2}\left(\theta_{2}\right)$ for different $\beta_{1}=0.01,0.06 \ldots 0.51$ in case of no pairs. Each curve corresponds to a given $\beta_{1}$; different points on the curve correspond to different $n_{1}$. Only lower parts of the curves correspond to shock transitions. For each $\theta_{2}<\theta_{\max }$ the final state is reached via vertical isothermal jump between points on the curves.
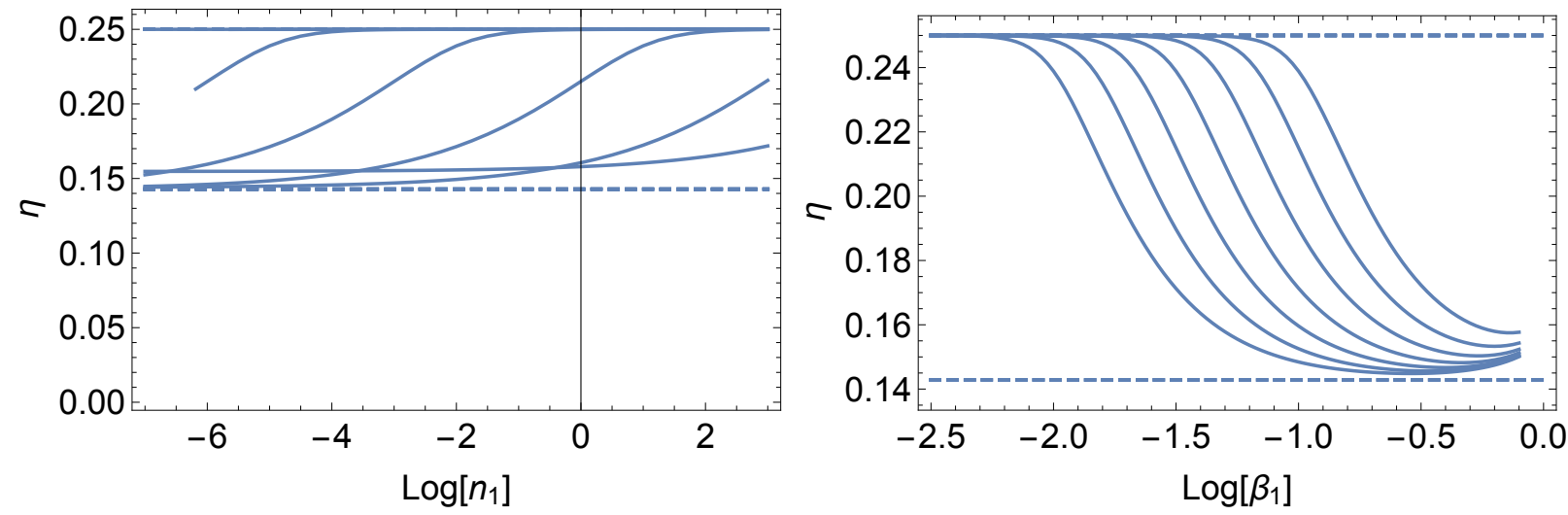

Fig. 5.- Compression ratio as function of shock velocity and upstream density; no pair creation. For high upstream density or low velocity the shock is matter-dominated with compression ratio approaching $1 / 4$, while for small density/high velocity the shock is radiation-dominated with compression ratio approaching 1/7. The compression ratio never goes above 7 .

\section{Stationary 1D flow with radiation and pair production}

After getting experience with simple cases we are ready to tackle the full problem: flow with radiation and pair production Let's assume that a shock propagates with velocity $\beta_{1} \leq 1$ through a cold plasma with ion density $\rho_{1}$. At each point one-dimensional, stationary non-relativistic flow is described by the following set of equations (mass, momentum and energy flux conservation)

$$
\begin{aligned}
& \beta_{1} \rho_{1}=\beta \rho \\
& \rho_{1} \beta_{1}^{2}=p_{t o t}+\rho_{t o t} \beta^{2} \\
& \rho_{1} \beta_{1}^{3} / 2=\left(w_{t o t}+\rho_{t o t} \beta^{2} / 2\right) \beta+F_{r}
\end{aligned}
$$



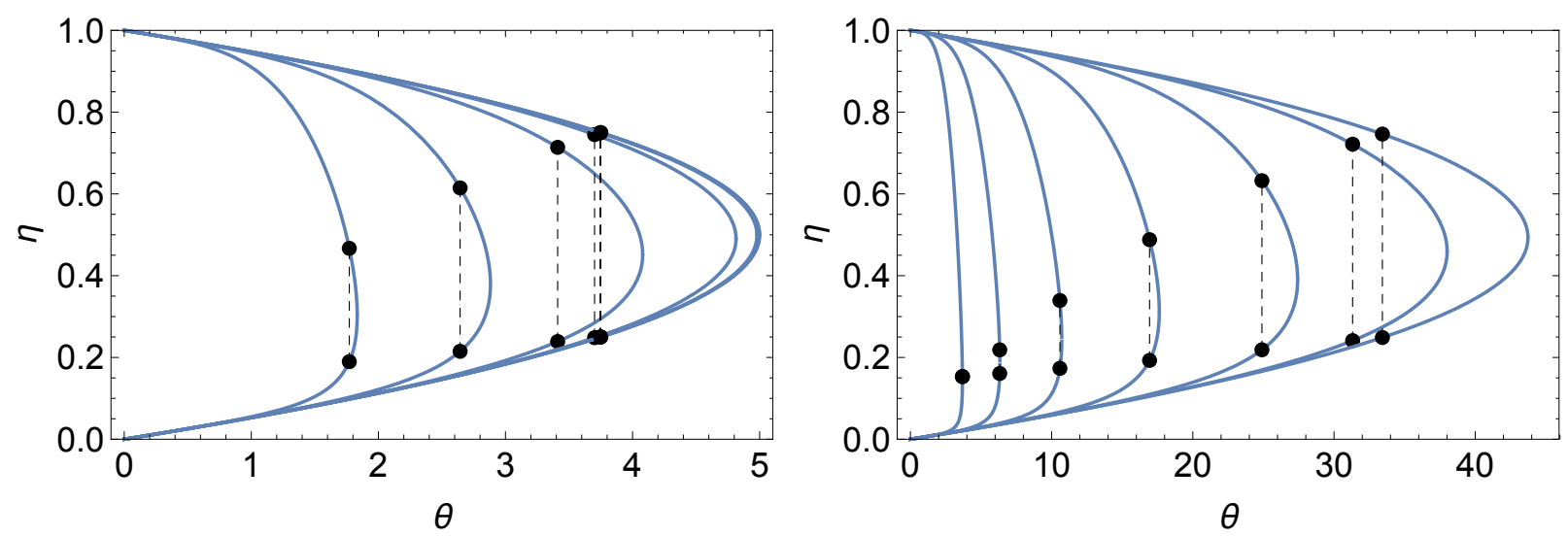

Fig. 6. - Evolution of flow without pair production on $\eta-\theta$ diagram. The flow evolves from the initial point $\eta=1, \theta=0$, the final temperature is reached at smaller compression ratios, upper dots. The isothermal jump forms, dashed lines. Different curves correspond to different densities, $n=10^{-1}, 1 \ldots 10^{5}$. Left panel: $\beta_{1}=0.1$, right panel $\beta_{1}=0.3$. For sufficiently small densities the isothermal jump disappears.

In $(33)$

$$
\rho_{\text {tot }}=\rho+n_{ \pm} m_{e}+u_{\text {rad }} / c^{2}
$$

is the total mass density that includes ion density $\rho$, pair mass density $n_{ \pm} m_{e}$ and effective mass density of radiation $u_{\text {rad }} / c^{2}$ ( $u_{\text {rad }}$ is the energy-density of thermal radiation),

$$
w_{2}=\frac{4}{3} u_{r a d}+\frac{\gamma}{\gamma-1} \frac{\rho}{m_{p}} T+n_{ \pm}\left(m_{e} c^{2}+4 T\right)
$$

is the plasma enthalpy not including ion rest mass, that includes contribution from radiation, ion energy density, pair rest mass and pair energy density, $\gamma=5 / 3$ is the adiabatic index of the ion component (assumed non-relativistic), the electron component is assumed to be relativistic (factor of 4 in the expression for the pair contribution). Finally, $F_{r}$ is the energy flux carried by radiation. We assume high optical depth limit, so that locally

$$
\begin{aligned}
& F_{r}=-\frac{c}{3 n_{t o t} \sigma_{T}} \nabla u_{r a d} \\
& u_{r a d}=\frac{4}{c} \sigma_{S B} T^{4}
\end{aligned}
$$

where total density has contributions both from neutralizing electrons and pairs, $n_{\text {tot }}=\rho / m_{p}+n_{ \pm}$.

We also make non-relativistic approximation, neglecting $\beta_{1}^{2} \ll 1$, but not when it comes with 
$\mu \gg 1$. The momentum and energy conservation equations give

$$
\begin{aligned}
& 45 \pi^{3 / 2} n_{1}\left(\theta-\beta_{1}^{2}(1-\eta) \eta \mu\right)+45 \sqrt{2} \eta \sqrt{g_{E}} e^{-1 / \theta} \theta^{5 / 2}+\pi^{5 / 2} \eta \theta^{4}=0 \\
& \frac{3 \pi^{3 / 2} \eta \theta^{3} \lambda_{C}}{\beta_{1} \alpha_{f}^{2}} \partial_{x} \theta=\left(\sqrt{2} \eta g_{e} e^{-1 / \theta} \theta^{3 / 2}+\pi^{3 / 2} n_{1}\right) \times \\
& \left(\frac{90 \sqrt{2} \eta g_{e} e^{-1 / \theta}(4 \theta+1) \theta^{3 / 2}}{\pi^{3 / 2}}+8 \pi \eta \theta^{4}+\frac{45 n_{1}\left(\beta_{1}^{2}(\gamma-1)\left(\eta^{2}-1\right) \mu+2 \gamma \theta\right)}{\gamma-1}\right)
\end{aligned}
$$

In the momentum equation terms are plasma contribution, pairs and radiation consequently.

Setting $\partial_{x} \theta=0$ in (38) determines the overall shock jump conditions. Since (38) is linear in $n_{1}$, we can eliminate $n_{1}$ and find how the compression ratio far downstream depends on the final temperature (for fixed $\beta_{1}$ ), Fig. 7

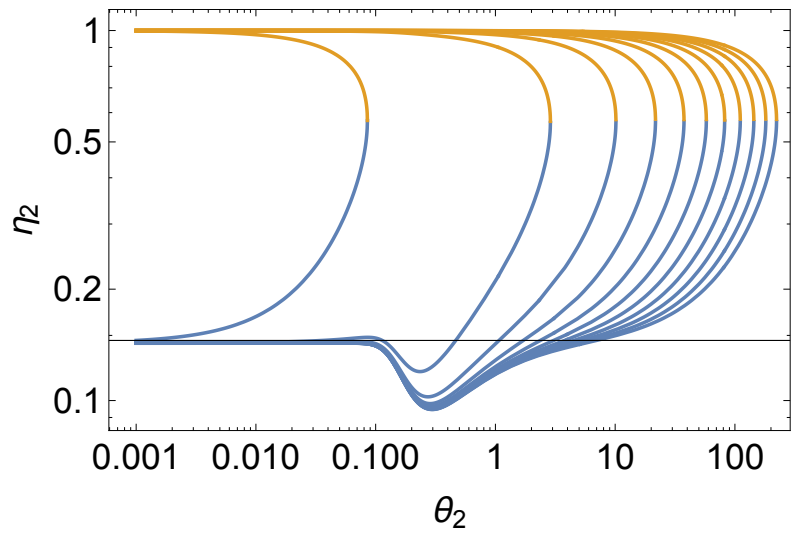

Fig. 7.- Dependence of $\eta_{2}\left(\theta_{2}\right)$ for different $\beta_{1}=0.01,0.06 \ldots 0.51$. Each curve corresponds to a given $\beta_{1}$; different points on the curve correspond to different $n_{1}$. Only lower parts of the curves correspond to shock transitions. For each $\theta_{2}<\theta_{\max }$ the final state is reached via vertical isothermal jump between points on the curves.

Few points are worth mentioning. At mild post-shock conditions the compression ratio can go below $1 / 7$ and reach $\sim 1 / 10$. This fairly mild modification is due to the formation of pairs - energy is spent on creating mass, not pressure. The effect is fairly mild since at smaller temperature there are few pairs, while at larger temperature pairs behave like radiation, so that their mass is not important. There is also a limiting case for large $n_{1}$, where pairs and radiation are not important, see (14).

The shock jump conditions are plotted in Fig. 8, where the final compression ration $\eta_{2}$ and the final temperature $\theta_{2}$ are plotted as functions of the upstream density and shock velocity

Evolution of quantities within the shock in $\eta-\theta$ plane are pictured in Figs. 9 and 10. Each panel is for a given velocity $\beta_{1}$ with different curves corresponding to different densities. For sufficiently small $n_{1}$ the isothermal jump disappears. We know that when the post-shock pressure 

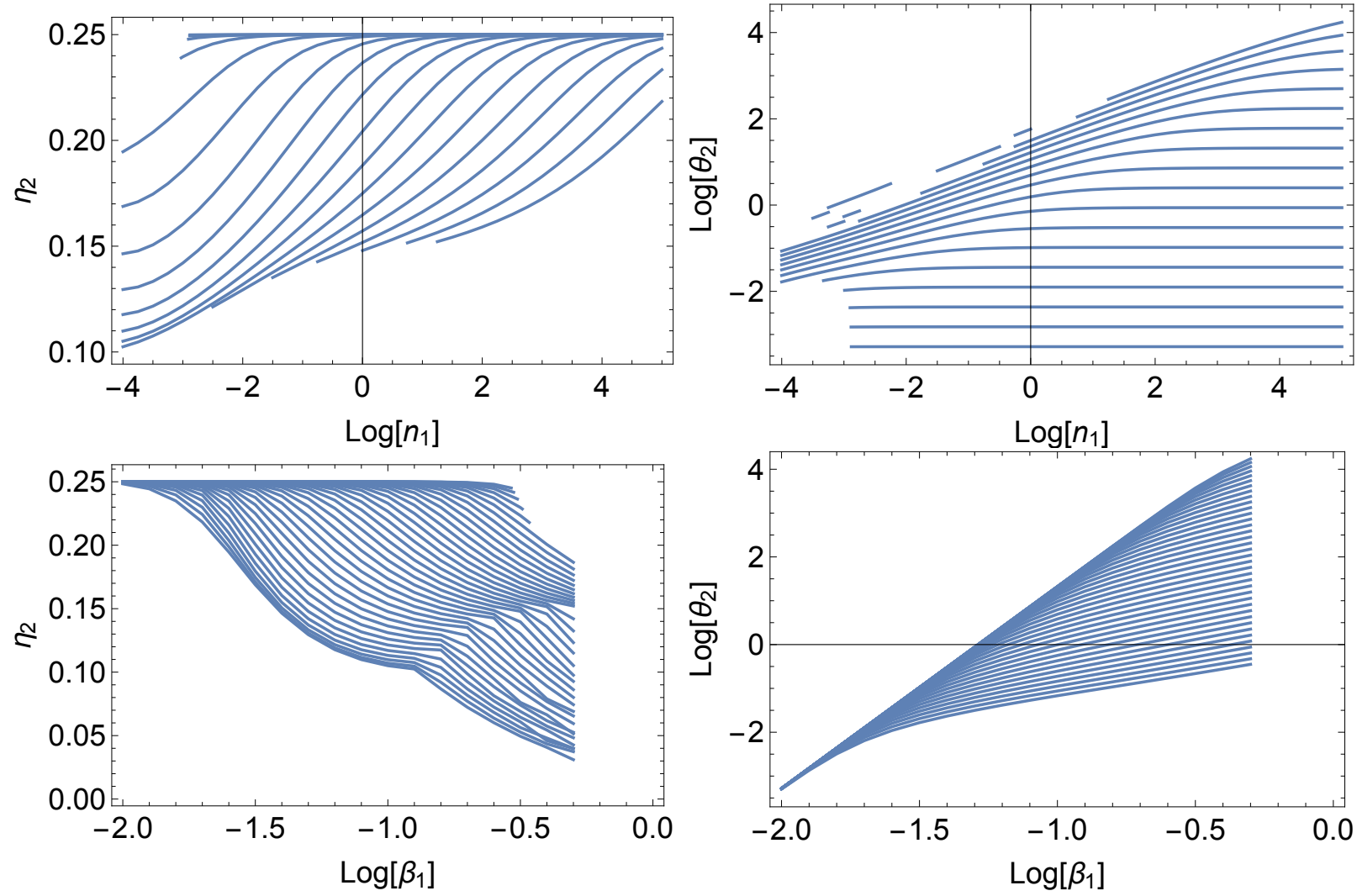

Fig. 8.- Jump conditions $\eta_{2}$ and $\theta_{2}$ for pair and radiative loaded shocks as function of upstream density for different shock velocities (top row, for $\beta_{1}=10^{-2}, 10^{-1.75}, \ldots, 10^{-0.25}$ ) and as function of shock velocity for different upstream density (bottom row, $n_{1}=10^{-4}, 10^{-3.75}, \ldots 10^{4}$ ).
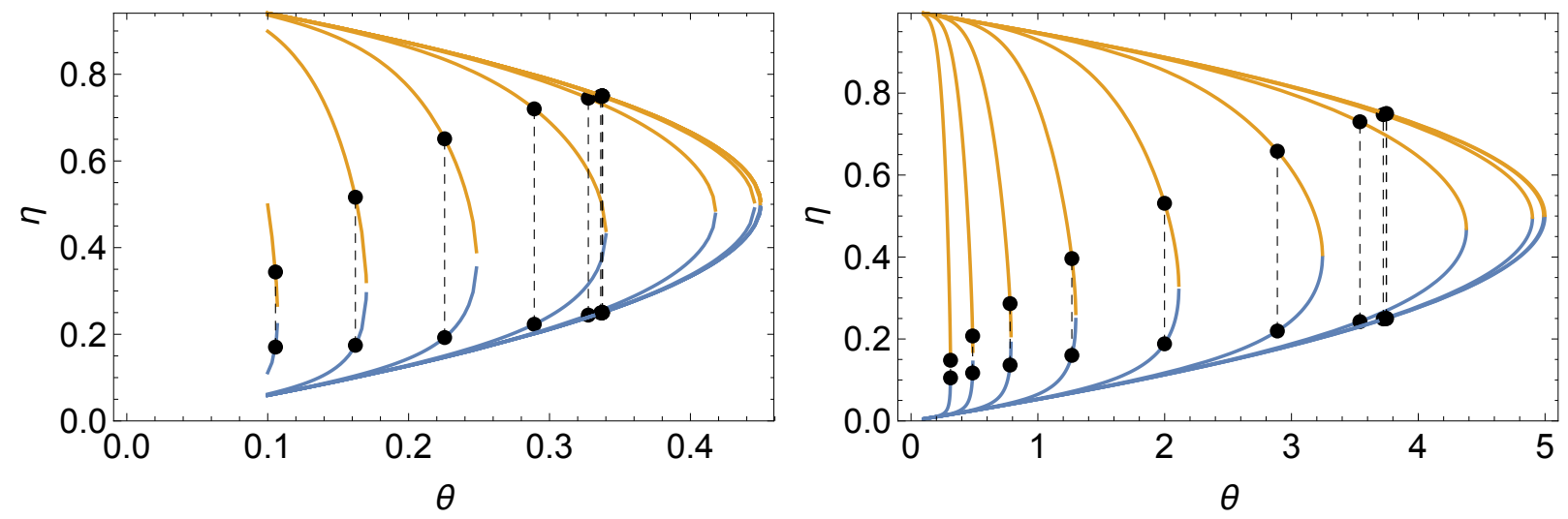

Fig. 9.- Evolution of pair and radiative loaded flows on $\eta-\theta$ plane for $\beta_{1}=0.03,0.1, n_{1}=$ $10^{-4}, 10^{-3} \ldots 10^{5}$.

and enthalpy are dominated by radiation, there is no isothermal jump - see $\S 4.1$. Analytically this can be seen from Eq. (37) where the only possibly negative term is proportional to density $n_{1}$. 


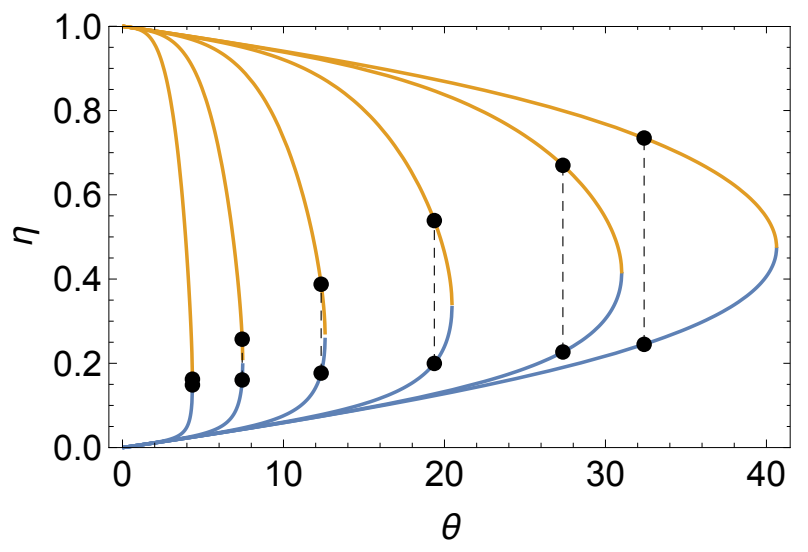

Fig. 10.- Evolution of pair and radiative loaded flows on $\eta-\theta$ plane for $\beta_{1}=0.3,0.1, n_{1}=$ $1,10 \ldots 10^{5}$. For small densities the isothermal jump disappears.

Numerical solutions give the following conditions for the disappearance of the isothermal jump, Fig. 11.

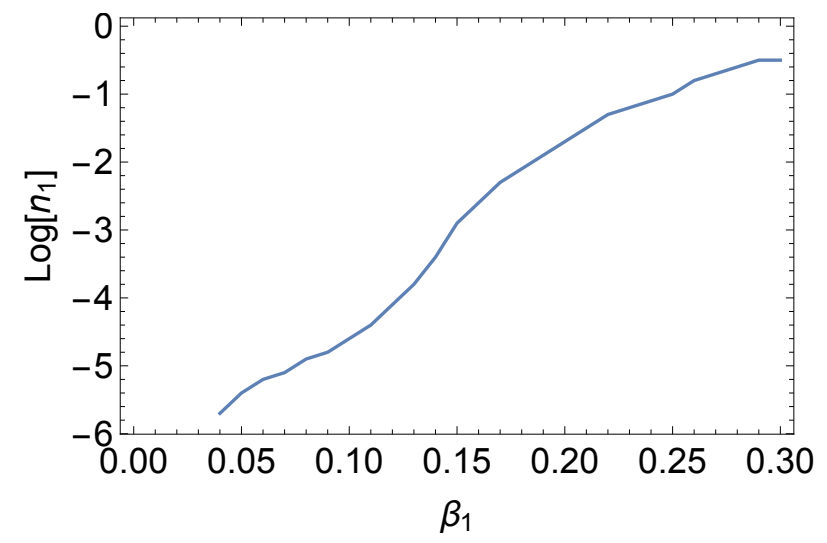

Fig. 11. - Condition for the appearance of the isothermal jump. For a given shock velocity the isothermal jump appears for densities above the curve.

\section{Discussion}

In this paper we address the structure of pair- and radiation-loaded shock transitions. The first most important effect is the energy redistribution within the flow due to diffusive propagation of radiation. This generically leads to the formation of a special surface within the flow - the isothermal jump. (The isothermal jump is different from highly radiative isothermal shocks; in our case the flow is energy conserving.) The isothermal jump forms first for plasma parameters when energy density of radiation and pair can be neglected, yet energy redistribution within the flow changes the flow properties qualitatively - even small energy density of radiation increases the 
order of the governing differential equation and thus cannot be neglected.

For increasing post-shock temperature the role of radiation pressure increases with respect to plasma pressure. As a result, for sufficiently strong shocks the isothermal jump disappears - highly radiation-dominated shocks smoothly reach the final state.

Overall, formation of pairs has only a mild effect on the shock structure in the regime $\theta \sim 1$. This is due to the fact that at sub-relativistic temperatures the number of pairs is exponentially suppressed, while at highly relativistic regime thermodynamically pairs behave similar to photons. But formation of pairs may be important for the diffusive approximation to apply.

I would like to thank Maxim Barkov and Amir Levinson for discussions and organizers of the workshop "Cosmic Accelerators" at the Joint Space-Science Institute where part of this work

has been performed. This work had been supported by NSF grant AST-1306672, DoE grant desc0016369 and NASA grant 80NSSC17K0757.

\section{REFERENCES}

Abbott, B. P., et al. 2017, ApJ, 848, L13

Bromberg, O., Tchekhovskoy, A., Gottlieb, O., Nakar, E., \& Piran, T. 2017, ArXiv e-prints

Budnik, R., Katz, B., Sagiv, A., \& Waxman, E. 2010, ApJ, 725, 63

Gottlieb, O., Nakar, E., Piran, T., \& Hotokezaka, K. 2017, ArXiv e-prints

Landau, L. D., \& Lifshitz, E. M. 1959, Fluid mechanics

Lazzati, D., López-Cámara, D., Cantiello, M., Morsony, B. J., Perna, R., \& Workman, J. C. 2017, ApJ, 848, L6

Pozanenko, A., et al. 2017, ArXiv:1710.05448

Svensson, R. 1984, MNRAS, 209, 175

Wandel, A., \& Yahil, A. 1979, A\&A, 72, 367

Zeldovich, Y. B., \& Raizer, Y. P. 2003, Physics of Shock Waves (Dover Publications Inc.) 


\section{A. Finite Mach number: resolving the precursor}

Equation (16) has a special point upfront of the shock, at $\eta=1$, where the derivative $\partial_{x} \eta$ diverges, implying that the very front of the shock is located at finite distance with non-analytical behavior. Finite upstream Mach number resolves this singularity, as we demonstrate next.

For finite upstream temperature

$$
\begin{aligned}
& \rho_{1} v_{1}=\rho v \\
& p_{1}+\rho_{1} v_{1}^{2}=p+\rho v^{2} \\
& v_{1}\left(\frac{1}{2} \rho_{1} v_{1}^{2}+w_{1}\right)=F_{r}+v\left(\frac{\rho v^{2}}{2}+w\right) \\
& F_{r}=-\frac{4 \pi^{2} c^{3} m_{e} \theta_{T}^{3}}{45 n \lambda_{C}^{3} \sigma_{T}} \partial_{x} \theta_{T}
\end{aligned}
$$

Mass and momentum conservation give

$$
\begin{aligned}
& \eta=\frac{ \pm \sqrt{\left(\theta_{T, 1}+\beta_{1}^{2} \mu\right)^{2}-4 \beta_{1}^{2} \mu \theta_{T}}+\theta_{T, 1}+\beta_{1}^{2} \mu}{2 \beta_{1}^{2} \mu} \rightarrow \frac{1}{2} \pm \frac{\sqrt{\beta_{1}^{2} \mu-4 \theta_{T}}}{2 \beta_{1} \sqrt{\mu}} \\
& \frac{1}{2}\left(1-\sqrt{1-\frac{\theta_{T}}{\theta_{T, \max }}}\right) \\
& \theta_{T}=\eta\left(\theta_{T, 1}-\beta_{1}^{2}(\eta-1) \mu\right) \rightarrow \beta_{1}^{2}(1-\eta) \eta \mu
\end{aligned}
$$

where the second relations indicate $\theta_{T, 1} \rightarrow 0$ limit.

Thus, there are two branches for $\eta(\theta)$ which connect at

$$
\begin{aligned}
& \eta_{\text {crit }}=\frac{1}{2}+\frac{\theta_{T, 1}}{2 \beta_{1}^{2} \mu} \rightarrow \frac{1}{2} \\
& \theta_{T, \max }=\frac{\left(\theta_{T, 1}+\beta_{1}^{2} \mu\right)^{2}}{4 \beta_{1}^{2} \mu} \rightarrow \frac{\beta_{1}^{2} \mu}{4}
\end{aligned}
$$

The equation for the compression ratio becomes

$$
\frac{\pi \beta_{1}^{5}(\gamma-1) \mu^{3}}{15 \alpha_{f}^{2}{ }^{2} 1^{2} \lambda_{C}^{5}} \partial_{x} \eta=\frac{\beta_{1}^{6}(\eta-1) \mu^{3}\left(-2 \gamma \theta_{T, 1}+\beta_{1}^{2} \gamma(\eta-1) \mu+\beta_{1}^{2}(\eta+1) \mu\right)}{\eta^{4}\left(\beta_{1}^{2}(\eta-1) \mu-\theta_{T, 1}\right)^{3}\left(\theta_{T, 1}+\beta_{1}^{2}(1-2 \eta) \mu\right)} \rightarrow \frac{\gamma(\eta-1)+\eta+1}{(1-2 \eta)(1-\eta)^{2} \eta^{4}}
$$

For finite $\theta_{T, 1}$ the compression ratio far upstream,

$$
\eta \propto e^{\frac{30 \beta_{1} n_{1}^{2} z \lambda_{C}^{5} \alpha_{f}^{2}}{\pi(\gamma-1) \theta_{T, 1}^{3}}}
$$

is finite for all $x$ (upstream medium corresponds to $x<0$ ). For small upstream temperature the bulk of the transition is well described by the $\theta_{T, 1}=0$ limit. 


\section{B. Properties of the isothermal jump}

Next, at the iso-thermal jump the sound speed is

$$
c_{s}=\sqrt{\gamma T / m_{p}}=\frac{\sqrt{2} \sqrt{\gamma-1} \sqrt{\gamma}}{\gamma+1} v_{1}
$$

At this point, on the upper branch the parameters of the flow are

$$
\begin{aligned}
& \eta_{+}=2 /(\gamma+1) \\
& v_{+}=\frac{2}{\gamma+1} v_{1}=\frac{3}{4} v_{1} \\
& M_{+}=\frac{\sqrt{2}}{\sqrt{\gamma-1} \sqrt{\gamma}}=\frac{3}{\sqrt{5}}
\end{aligned}
$$

While in the post-jump flow

$$
\begin{aligned}
& \eta_{2}=\frac{\gamma-1}{\gamma+1} \\
& v_{2}=\frac{\gamma+1}{\gamma-1} v_{1}=\frac{1}{4} v_{1} \\
& M_{2}=\frac{\sqrt{\gamma-1}}{\sqrt{2 \gamma}}=\frac{1}{\sqrt{5}}
\end{aligned}
$$

The compression ratio at the isothermal jump is $(\gamma-1) / 2=1 / 3$. 